UMTRI-2012-19

JULY 2012

\title{
Toward Designing Pedestrian-Friendly VEHICLES
}

\author{
JINGWEN HU \\ KATHLEEN D. KLINICH
}

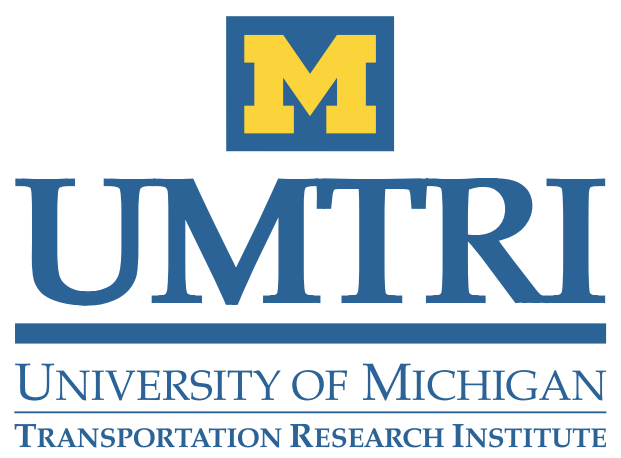




\title{
TOWARD DESIGNING PEDESTRIAN-FRIENDLY VEHICLES
}

\author{
Jingwen $\mathrm{Hu}$ \\ Kathleen D. Klinich
}

The University of Michigan

Transportation Research Institute

Ann Arbor, Michigan 48109-2150

U.S.A.

Report No. UMTRI-2012-19

July 2012 
Technical Report Documentation Page

\begin{tabular}{|l|l|l|}
\hline 1. Report No. & 2. Government Accession No. & 3. Recipient's Catalog No. \\
\hline $\begin{array}{l}\text { 4. Title and Subtitle } \\
\text { Toward Designing Pedestrian-Friendly Vehicles }\end{array}$ & $\begin{array}{l}\text { 5. Report Date } \\
\text { July } 2012\end{array}$ \\
\cline { 2 - 2 } & $\begin{array}{l}\text { 6. Performing Organization Code } \\
383818\end{array}$ \\
\hline $\begin{array}{l}\text { 7. Author(s) } \\
\text { Jingwen Hu and Kathleen D. Klinich }\end{array}$ & $\begin{array}{l}\text { 8. Performing Organization Report No. } \\
\text { UTRI-2012-19 }\end{array}$ \\
\hline $\begin{array}{l}\text { 9. Performing Organization Name and Address } \\
\text { The University of Michigan }\end{array}$ & 10. Work Unit no. (TRAIS) \\
2901 Baxter Road, Ann Arbor, Michigan 48109-2150 U.S.A. & 11. Contract or Grant No. \\
\hline $\begin{array}{l}\text { 12. Sponsoring Agency Name and Address } \\
\text { The University of Michigan } \\
\text { Sustainable Worldwide Transportation }\end{array}$ & 13. Type of Report and Period \\
\hline
\end{tabular}

The current members of Sustainable Worldwide Transportation include Autoliv Electronics, China FAW Group, General Motors, Honda R\&D Americas, Meritor WABCO, Michelin Americas Research, Nissan Technical Center North America, Renault, Saudi Aramco, Toyota Motor Engineering and Manufacturing North America, and Volkswagen Group of America. Information about Sustainable Worldwide Transportation is available at: http://www.umich.edu/ umtriswt

16. Abstract

Due to a rise in global urbanization and motorization, pedestrian injuries continue to be a major public-health problem worldwide. To design pedestrian-friendly vehicles, knowledge of common pedestrian injuries, technologies available for reducing these injuries, and benefit estimations for these technologies are essential. In this report, we present a literature review and provide insights into vehicle designs for improving pedestrian safety.

Pedestrian-injury data show that heads and lower extremities are the most commonly injured body regions in vehicle-to-pedestrian crashes, but chest injuries are the second most common injuries after head injuries to cause fatalities. Pedestrian injuries are strongly related to impact speed, pedestrian age, and vehicle type. The increased proportion of older pedestrians and SUVs will likely result in more pedestrian injuries, especially those involving the torso.

Adding energy-absorbing materials to the vehicle front-end structures is the most cost-effective way of developing pedestrian-friendly vehicles. However, such improvements often conflict with other design considerations, such as styling, aerodynamics, and safety standards for low-speed crashes and rollovers. Deployable passive safety designs, such as pop-up hoods and windshield airbags, and active safety designs, such as brake-assist systems and autonomous-braking systems, have demonstrated considerable benefits for reducing pedestrian injuries. Integrated passive and active systems are recommended for a further enhancement of pedestrian protection. However, the benefits from different pedestrian-safety designs vary with different types of vehicles and pedestrians with different statures and ages. Consequently it is important to consider implementing specific safety designs to a specific vehicle for maximizing the effectiveness, and population-age profile may also play an important role in selecting pedestrian safety features.

\begin{tabular}{|c|c|c|c|}
\hline \multicolumn{3}{|c|}{$\begin{array}{l}\text { 17. Key Words } \\
\text { Pedestrian Injuries, Vehicle Design, Design Benefits }\end{array}$} & $\begin{array}{l}\text { 18. Distribution Statement } \\
\text { Unlimited }\end{array}$ \\
\hline $\begin{array}{l}\text { 19. Security Classification (of this report) } \\
\text { None }\end{array}$ & $\begin{array}{l}\text { 20. Security Classification (of this page) } \\
\text { None }\end{array}$ & $\begin{array}{l}\text { 21. No. of Pages } \\
51\end{array}$ & 22. Price \\
\hline
\end{tabular}




\section{Acknowledgments}

This research was supported by Sustainable Worldwide Transportation (http://www.umich.edu/ umtriswt). The current members of Sustainable Worldwide Transportation include Autoliv Electronics, China FAW Group, General Motors, Honda R\&D Americas, Meritor WABCO, Michelin Americas Research, Nissan Technical Center North America, Renault, Saudi Aramco, Toyota Motor Engineering and Manufacturing North America, and Volkswagen Group of America.

The authors would like to thank Michael Sivak and Robert Sweet from UMTRI for their comments on this report. 


\section{Contents}

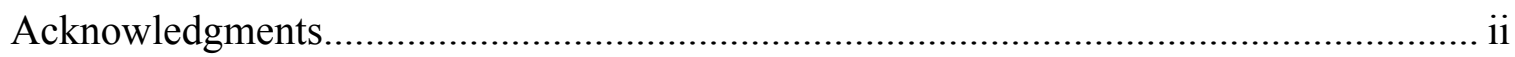

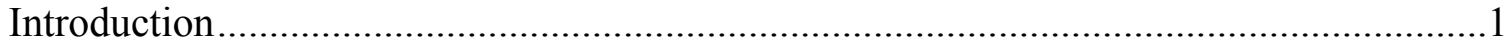

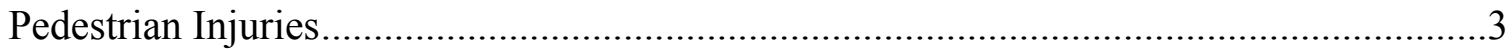

Sequence of Events in a Vehicle-to-Pedestrian Crash .....................................................

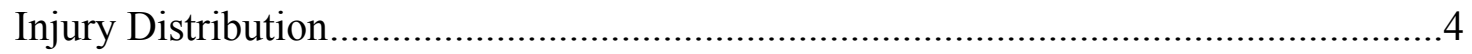

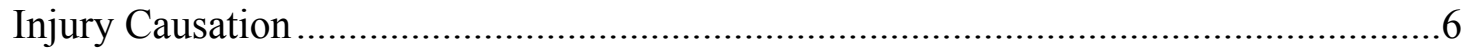

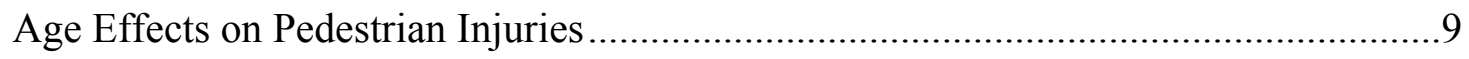

Pedestrian Injury Risk as a Function of Impact Speed .................................................11

Vehicle Type Effects on Pedestrian Injuries.............................................................13

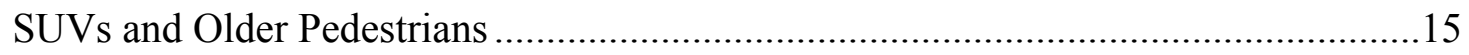

Test Procedures for Pedestrian Protection ...................................................................18

Euro NCAP Pedestrian Protection Test Procedure ....................................................18

Limitations of Current Pedestrian Impact-Testing Procedures ......................................20

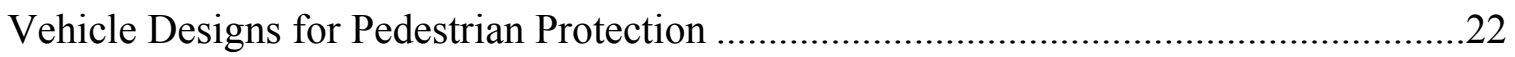

Basic Design Theories of Pedestrian-Friendly Vehicles.............................................22

Designs for Reducing Pedestrian Lower-Extremity Injuries ........................................23

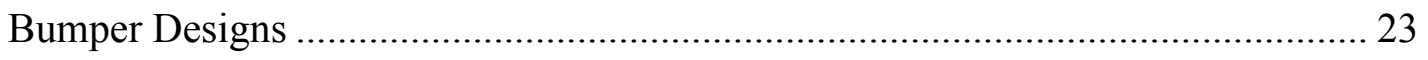

The Hood Leading-Edge Designs .................................................................... 26

Designs for Reducing Pedestrian Head Injuries ...................................................2

Hood and Pop-up Hood Designs ............................................................................. 27

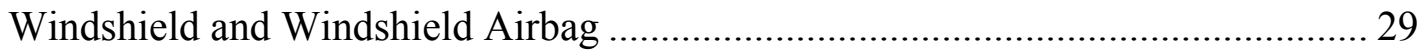

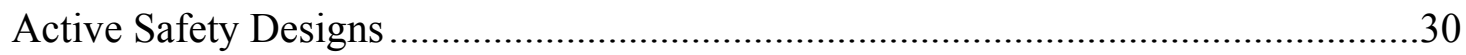

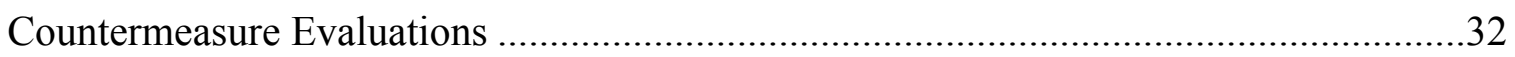

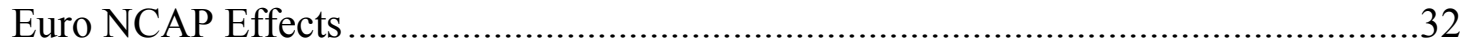

Benefit Estimation for Passive and Active Countermeasures.......................................32

Factors Affecting the Benefits from Countermeasures for Pedestrian Protection .........33

Interactions among Pedestrian Safety Designs........................................................ 34

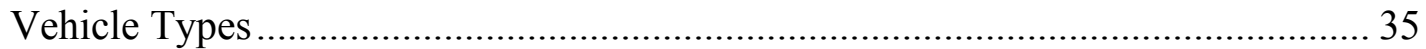

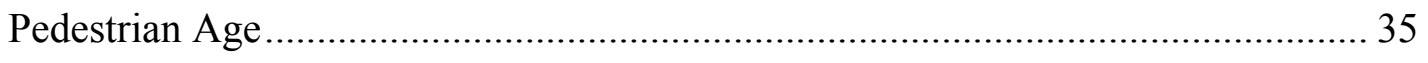

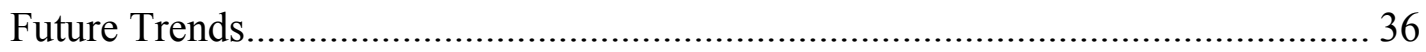




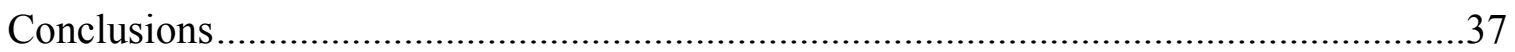

Pedestrian Injury Distribution, Causations, and Risk Factors ....................................37

Pedestrian Safety Designs and Benefit Estimation .................................................37

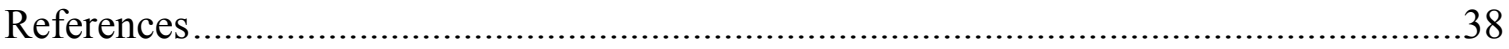




\section{Introduction}

Due to the rise in global urbanization and motorization, pedestrian injuries continue to be a major public-health problem worldwide. Every year over 1.2 million people die and 20 to 50 million people are injured in motor-vehicle crashes around the world, and pedestrians account for more than a third of them (WHO 2009). The proportion of pedestrians among road-traffic fatalities varies significantly in different countries (Figure 1), ranging from more than half in Africa to $15 \%$ or less in North America or Europe (Naci et al. 2009). The distribution also varies across countries with different income levels: $45 \%$ of road fatalities in low-income countries are pedestrians, whereas an estimated $29 \%$ of road fatalities in middle-income and $18 \%$ in high-income countries are pedestrians (Naci et al. 2009). As an interdisciplinary research problem, reducing pedestrian injuries needs a systematic approach, including safer facilities for pedestrians through engineering, safety laws with stronger enforcement, and higher public awareness through education (Zegeer and Bushell 2012). Historically, the benefit of designing a pedestrian-friendly vehicle was neglected primarily due to the societal perception that pedestrians are too vulnerable in a collision with a vehicle (Fisher and Hall 1972; Crandall et al. 2002). However, since the 1970s, and especially during the last two decades, field data analyses, pedestrian crash tests, and computational simulations have all shown that vehicle design has significant effects on pedestrian injuries.

This report provides insights into vehicle design and new vehicle-safety devices for improving pedestrian safety. The report starts with a summary of pedestrian-injury distribution, causation, and risk factors. A discussion follows on current pedestrian impact tests and the safety features specifically designed for reducing pedestrian head and lower-extremity injuries. Design evaluation and benefit are estimated for each safety feature. The report concludes with a discussion and recommendations. 


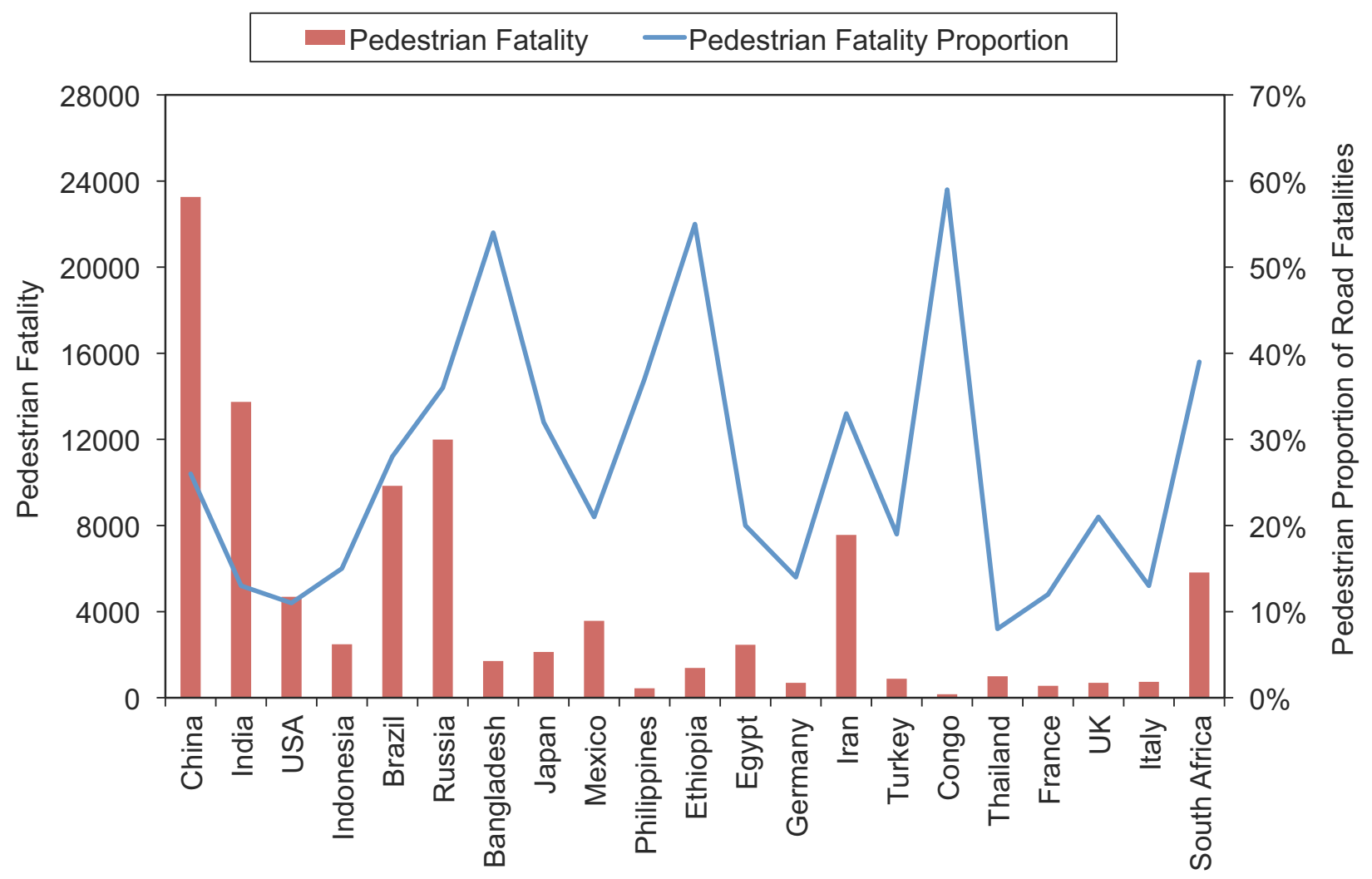

Figure 1. Pedestrian fatalities in countries with population more than 50 million (WHO 2009). (Data for Pakistan, Nigeria, and Vietnam were not available.) 


\section{Pedestrian Injuries}

Real-world pedestrian-injury data provide first-hand information about patterns, causation, and risk factors of pedestrian injuries, and valuable background for improving vehicle design for pedestrian protection. However, pedestrian accident databases with indepth investigation on both crashes and injuries are available for only a small number of developed countries. These include the U.S. Pedestrian Crash Data Study (PCDS) (Ivarsson et al. 2005; Zhang et al. 2008; Helmer et al. 2010), the German In Depth Accident Study (GIDAS) (Fredriksson et al. 2010; Otte et al. 2012), and the European In Depth Pedestrian Database APROSYS (Advanced PROtection SYStems) (Carter et al. 2008). The International Harmonized Research Activities Pedestrian Safety Working Group (IHRA/PS-WG) combined recent pedestrian-injury data from Japan, Germany, United States, and Australia, and denoted it as the IHRA Pedestrian Accident Dataset (Mizuno 2005). It is the most comprehensive dataset for pedestrian injuries thus far, but does not include data from developing countries. More recently, China has collected indepth pedestrian-accident data at a regional level (Kong and Yang 2010; Zhao et al. 2010), which provided an opportunity to compare pedestrian-injury rates among highincome and middle/low-income countries.

\section{Sequence of Events in a Vehicle-to-Pedestrian Crash}

Understanding the sequence of events in a vehicle-to-pedestrian collision is critical for vehicle designers. Fortunately, the chronology of such events has been well documented (Crandall et al. 2002). A vehicle-to-pedestrian crash generally starts with the bumper-to-leg contact, followed by the impact between the hood leading edge and the upper thigh or pelvis. Depending on the relative heights of the pedestrian and the vehicle front, the hood leading edge can hit the abdomen or chest as well. During the impact, the pedestrian often wraps around the front of the vehicle until the head and upper torso are struck by the top surface of the hood and/or windshield. The final impact in a pedestrian crash is almost always caused by the ground, but most pedestrian injuries occur due to the interaction with the vehicle (Crandall et al. 2002; Mizuno 2005; Helmer et al. 2010; Otte et al. 2012). Despite what seems to be a well-defined sequence of events, the precise trajectory and injuries of a pedestrian depend on many factors, including pedestrian 
factors (age, height, etc.), crash factors (impact speed, impact angle, etc.), and vehicle factors (vehicle type, stiffness, curvature, etc.).

\section{Injury Distribution}

As shown in Figure 2, even though the injury percentages per body region vary among different countries, they all show a consistent U-shape, in which the head and lower extremities are the most common injured body regions in pedestrians (Mizuno 2005; Yang 2005; Martin et al. 2011); the body regions in between are less susceptible to the risk of injuries.

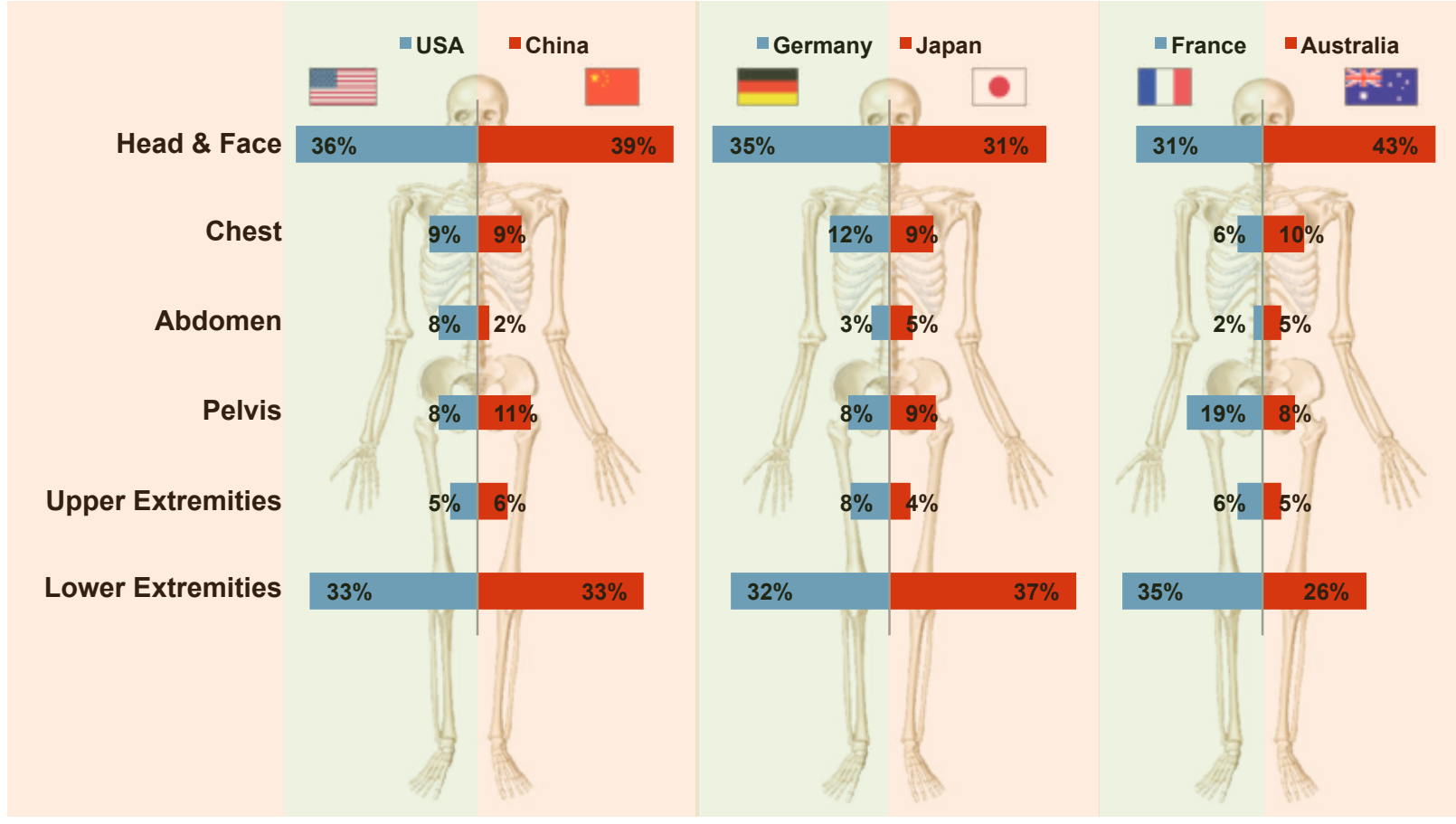

Figure 2. Percentage of AIS2+ injuries per body region in different countries (Mizuno 2005; Yang 2005; Martin et al. 2011).

Figure 2 provides a general view of pedestrian injuries using the Abbreviated Injury Scale (AIS) 2+. The AIS is an injury-measurement system that classifies an individual injury by body region according to its relative severity on the following 6point: scale 1 (minor), 2 (moderate), 3 (serious), 4 (severe), 5 (critical), and 6 (maximum) (AAAM 2008). The AIS ranks injuries with respect to their potential threat to life, but does not consider disability or cost factors. 
The majority of the AIS 2+ injuries are moderate (AIS 2) injuries, which have a low likelihood of fatality. To further explore the relationship between the frequency of injuries by body region and the severities of injuries, the percentage of pedestrians suffering from at least one injury of a given severity by body region is shown in Figure 3, using the pedestrian injury data from PCDS (Helmer et al. 2010). The increasing importance of head and torso (including chest, abdomen, and spine) injuries at higher AIS levels is clearly demonstrated. A study focusing on pedestrian torso injuries also found that torso injury is second only to head injury as the most important contributor to morbidity among adult pedestrians struck by light trucks and vans (LTVs), which include pickup trucks, minivans, and SUVs. Regardless of the striking vehicle type, torso injuries contribute to the overall cost of pedestrian morbidity at a level similar to lowerextremity injuries (Ivarsson et al. 2005). Although most previous research on pedestrian protection has focused on head and lower extremities, reducing torso-injury severity will also have high benefits for pedestrians, considering its high frequency among severe injuries.

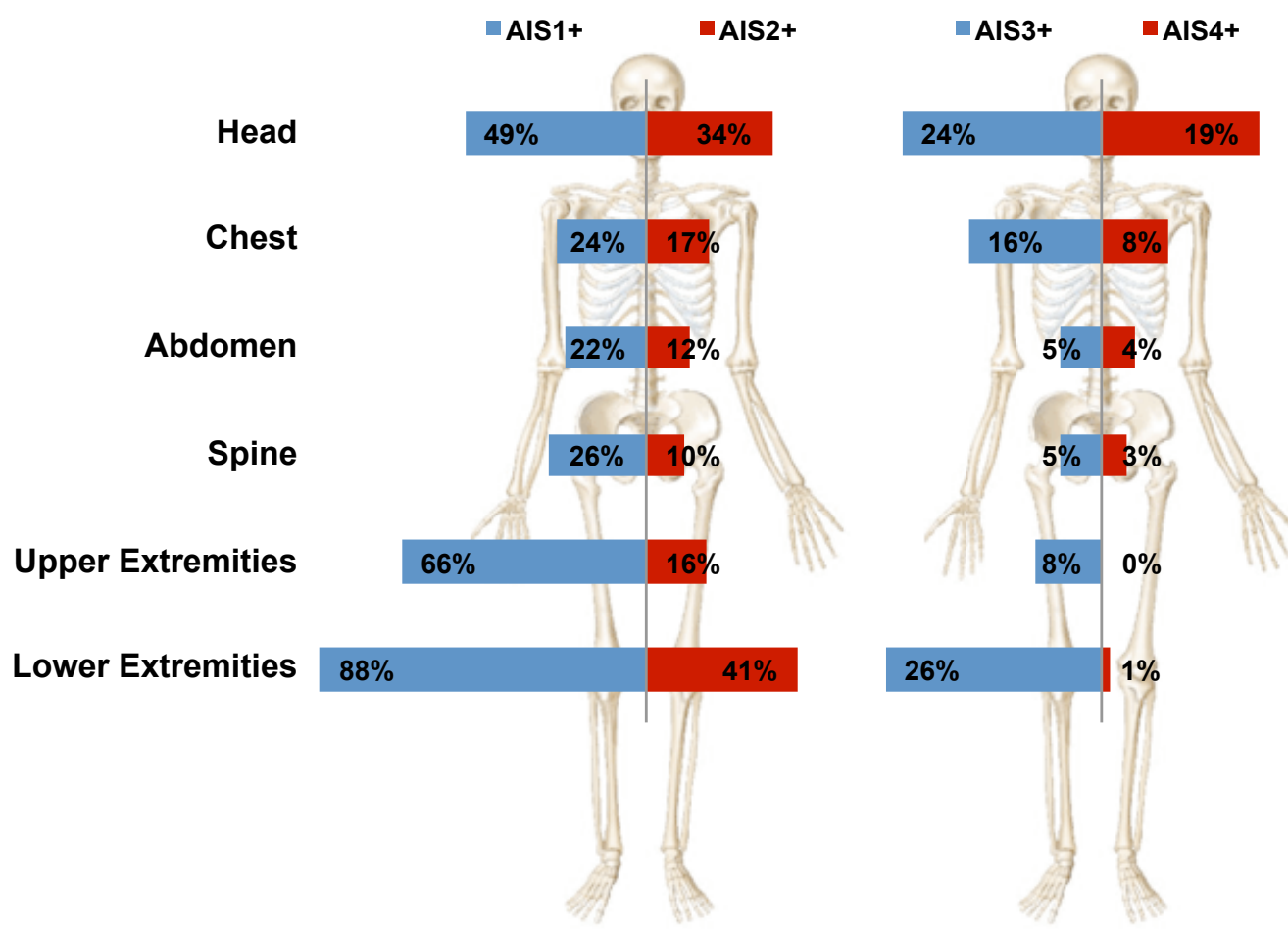

Figure 3. Probability of at least one injury to each body region by severity (Helmer et al. 2010). 


\section{Injury Causation}

The sources of pedestrian injuries have been well documented in the literature. Tables 1 to 3 summarize the sources of IHRA pedestrian AIS 2+ injuries for all ages, adults, and children, respectively (Mizuno 2005). Consistent with the sequence of impacts in a vehicle-to-pedestrian crash, the front bumper accounts for most lowerextremity injuries (61\%); the hood leading edge and hood are responsible for most pelvis (61\%), abdomen (69\%) and chest (54\%) injuries. The windshield, hood, and A-pillars account for a majority of head injuries (71\%). Children and adults show slightly different injury sources mainly because of differences in their stature. For example, head injuries for children are more commonly induced by the hood rather than the windshield and Apillar, the latter two being the most common injury sources for adult head injuries. Overall, vehicle components induced more than $85 \%$ of pedestrian injuries, highlighting the importance of designing pedestrian-friendly vehicles.

Table 1

Sources of IHRA pedestrian AIS 2+ injuries by body region, all age groups (Mizuno 2005).

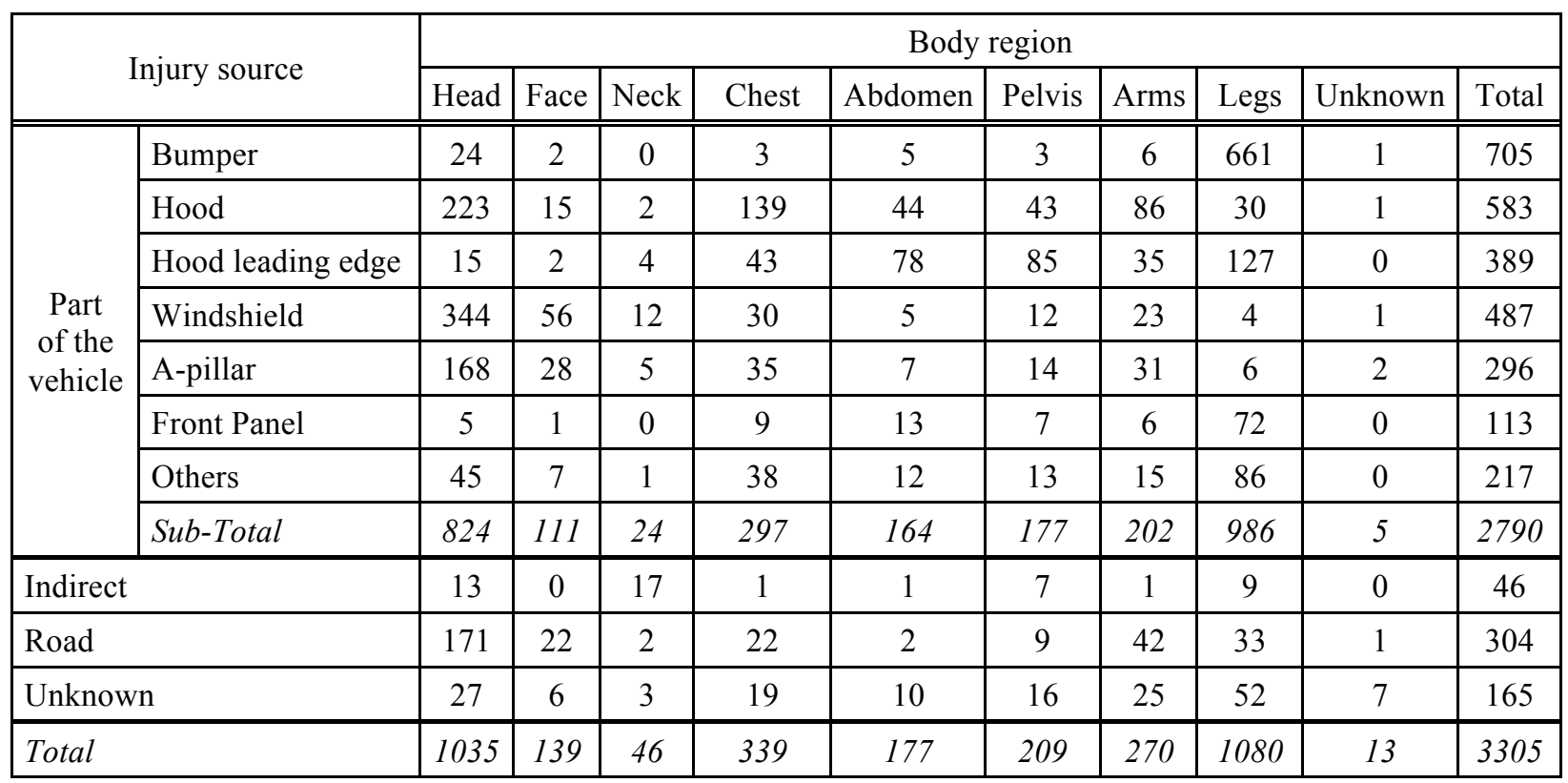


Table 2

Sources of IHRA pedestrian AIS 2+ injuries by body region, adults (age $>15$ )

(Mizuno 2005).

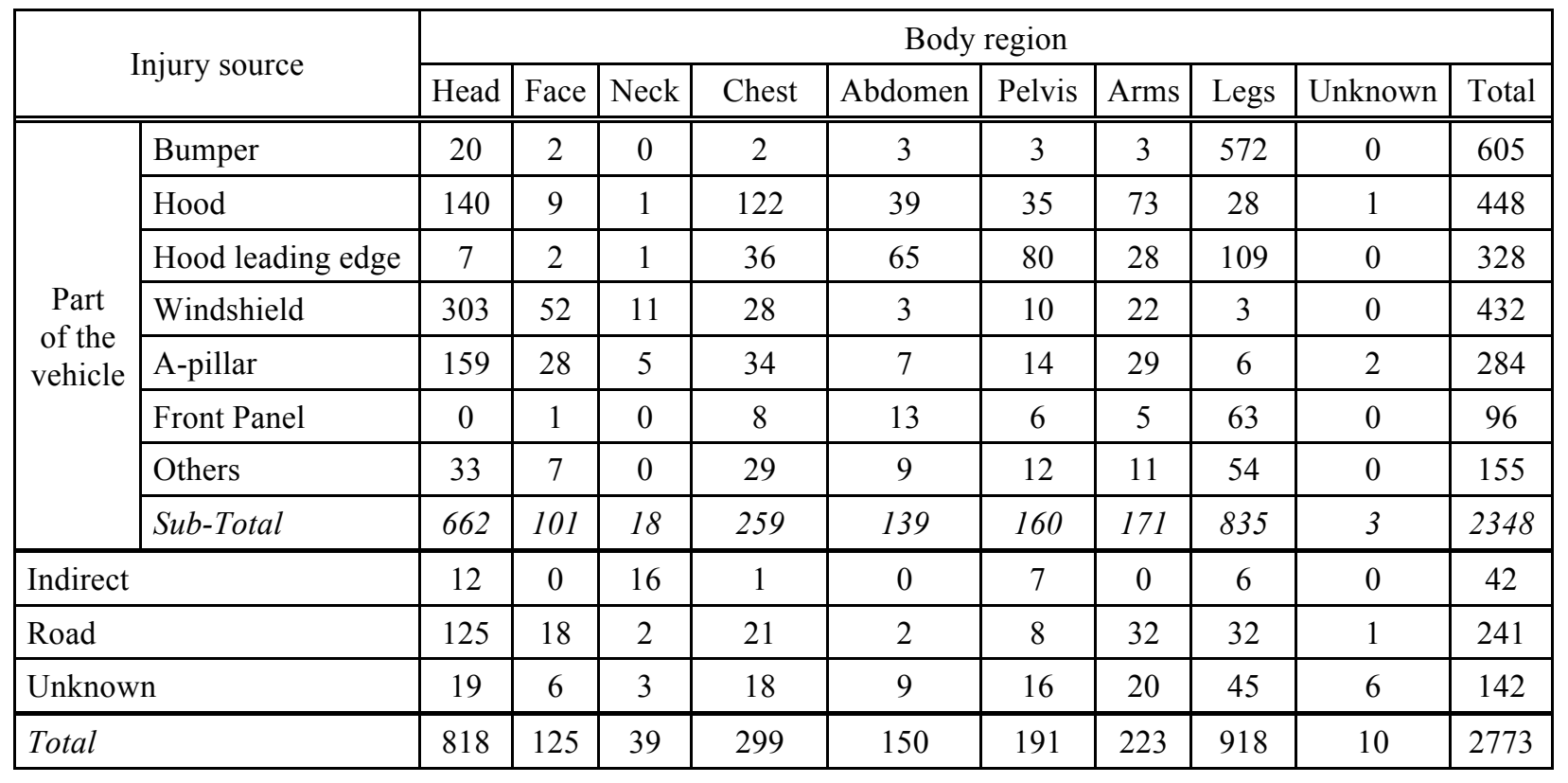

Table 3

Sources of IHRA pedestrian AIS $2+$ injuries by body region, children (age $\leq 15$ )

(Mizuno 2005).

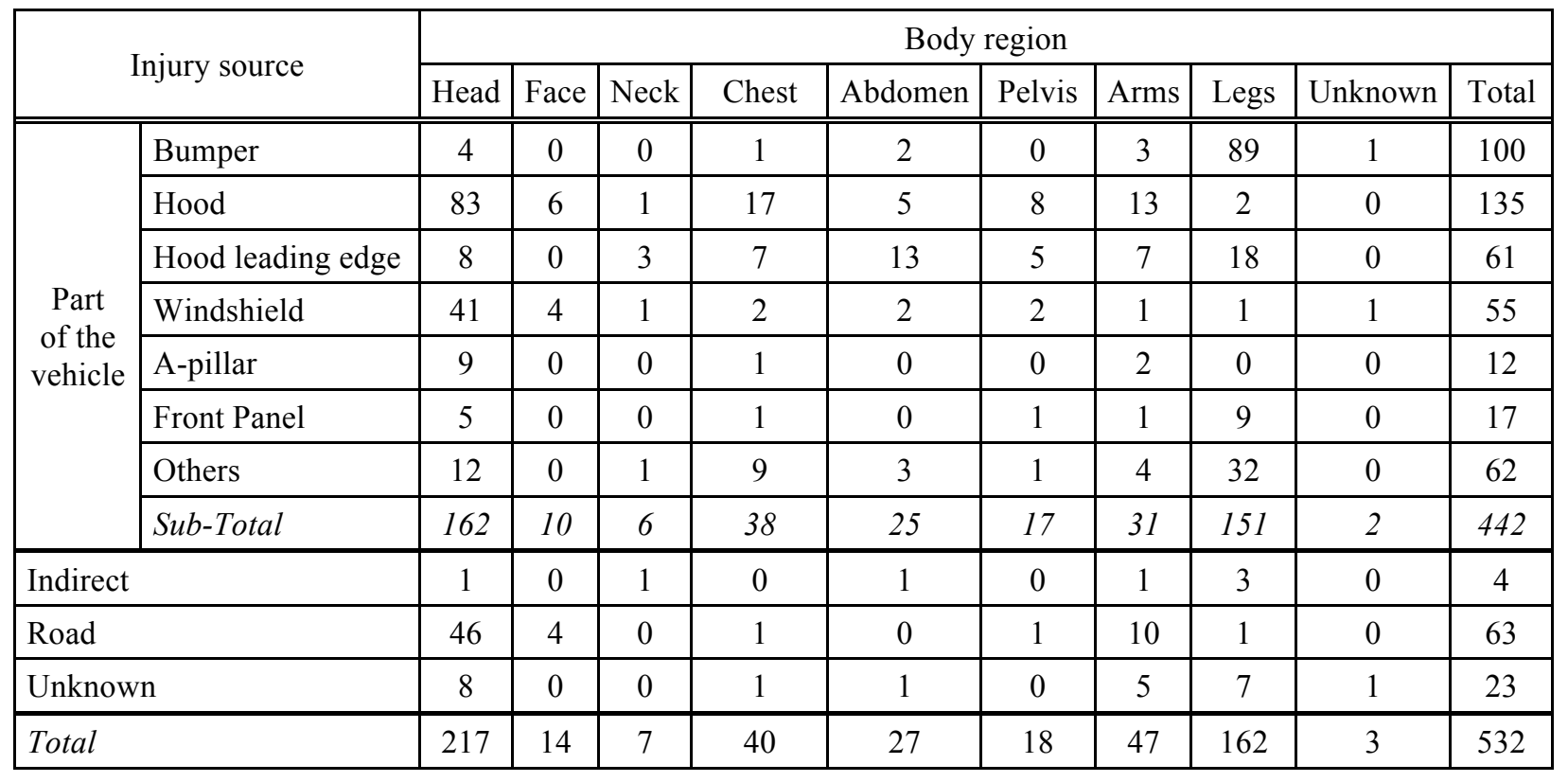


The IHRA study focused on AIS 2+ injuries, but reductions of low-severity injuries may not necessarily have a great impact on overall mortality. To identify particular vehicle components responsible for more severe injuries, Helmer et al. (2010) analyzed the relationship between the injury source and injury severity. This study found that the main components for AIS 4+ head and chest injuries are the windshield and hood; while the bumper is the main cause of AIS $3+$ lower-extremity injuries. Furthermore, this study found that the AIS level of head and chest injuries is hardly affected by vehicle impact region, but the AIS level of lower-extremity injuries is significantly affected by vehicle impact region. In particular, the hood edge was disproportionately represented in AIS 3+ lower-extremity injuries, indicating a potential redesign target.

Neal-Sturgess et al. (2007) used European pedestrian data from APROSYS to determine the injury sources most likely to cause fatal and nonfatal head injuries. They reported that fatal head injuries occurred predominantly on and around the windshield frame, including the A-pillars and cowl. This finding was confirmed by Richards et al. (2009), who found that impacts with the A-pillars caused proportionally more AIS 4+ head injuries than impacts with the windshield, based on data from London's Helicopter Emergency Medical Service (HEMS) and the UK's Police fatal data.

Targeting the societal cost of pedestrian injuries, Fildes et al. (2004) identified the priorities of vehicle-component and pedestrian-injury combinations using data on over 4,000 pedestrian crashes obtained from Australia and Germany. Windshield-to-head, bumper-to-lower-leg, hood-to-head, A-pillar-to-head, and hood-to-chest were found to be the top five priorities for adults; hood-to-head, bumper-to-upper-leg, front-area-to-head, bumper-to-lower-leg, and A-pillar-to-head were found to be the top five priorities for children. However, because this study focused on car and van collisions only, findings may not represent SUV or other light-duty vehicles.

To evaluate the association between vehicle type and the source and severity of pedestrian injuries, Roudsari et al. (2005) analyzed the PCDS data, and found that bumper-to-leg, windshield-to-head, hood-to-upper-extremity, and hood-to-chest were the leading sources for all injuries in pedestrian-to-passenger-car crashes; bumper-to-leg, hood-to-head, hood-edge-to-chest, and hood-to-chest were the leading sources for all 
injuries in pedestrian-to-LTV crashes. Overall, the most common source of AIS 3+ pedestrian injuries were windshield and hood for passenger cars, and hood edge and hood for LTVs.

Focusing only on AIS 3+ pedestrian injuries, Fredriksson et al. (2010) conducted an analysis using GIDAS data for 1998-2008. The most common injury sources for adult pedestrians were front-end-to-leg, windshield-to-head, hood-to-chest, and windshield-tochest; for children hood-to-head was the second most common injury source after frontend-to-leg. The high number of chest injuries is mainly due to the focus in this study on serious injuries only, suggesting the necessity for chest protection when designing pedestrian-friendly vehicles.

\section{Age Effects on Pedestrian Injuries}

Age is one of the most important pedestrian factors affecting injury risk in pedestrian crashes. Interestingly, the age effect on pedestrian injuries is nonlinear. Figure 4 shows that pediatric and elderly pedestrians are disproportionally common in vehicle-to-pedestrian crashes in France (Martin et al. 2011). IHRA data showed that children younger than 15 years old accounted for over $31 \%$ of all vehicle-to-pedestrian crashes in the U.S., Germany, Japan, and Australia, although they only accounted for $18 \%$ of the overall population in those countries. On the other hand, pedestrians under 15 years of age tend to sustain injuries with lower severities. Although older individuals did not show a high incidence rate in the IHRA data, they are more likely to suffer severe injuries in pedestrian crashes (Mizuno 2005). U.S. studies have also reported similar trends with children sustaining the highest incidence rate (Lee and Abdel-Aty 2005), and the elderly sustaining the highest severe-injury and fatality rate in pedestrian crashes (Demetriades et al. 2004; Henary et al. 2006; Kim et al. 2008). The high involvement of children in pedestrian crashes is largely a consequence of their lack of experience and safety awareness, as well as their small body size, which can make them more difficult to see; the high injury and fatality rate of the older population is mostly due to their agerelated morphological and physiological changes. 


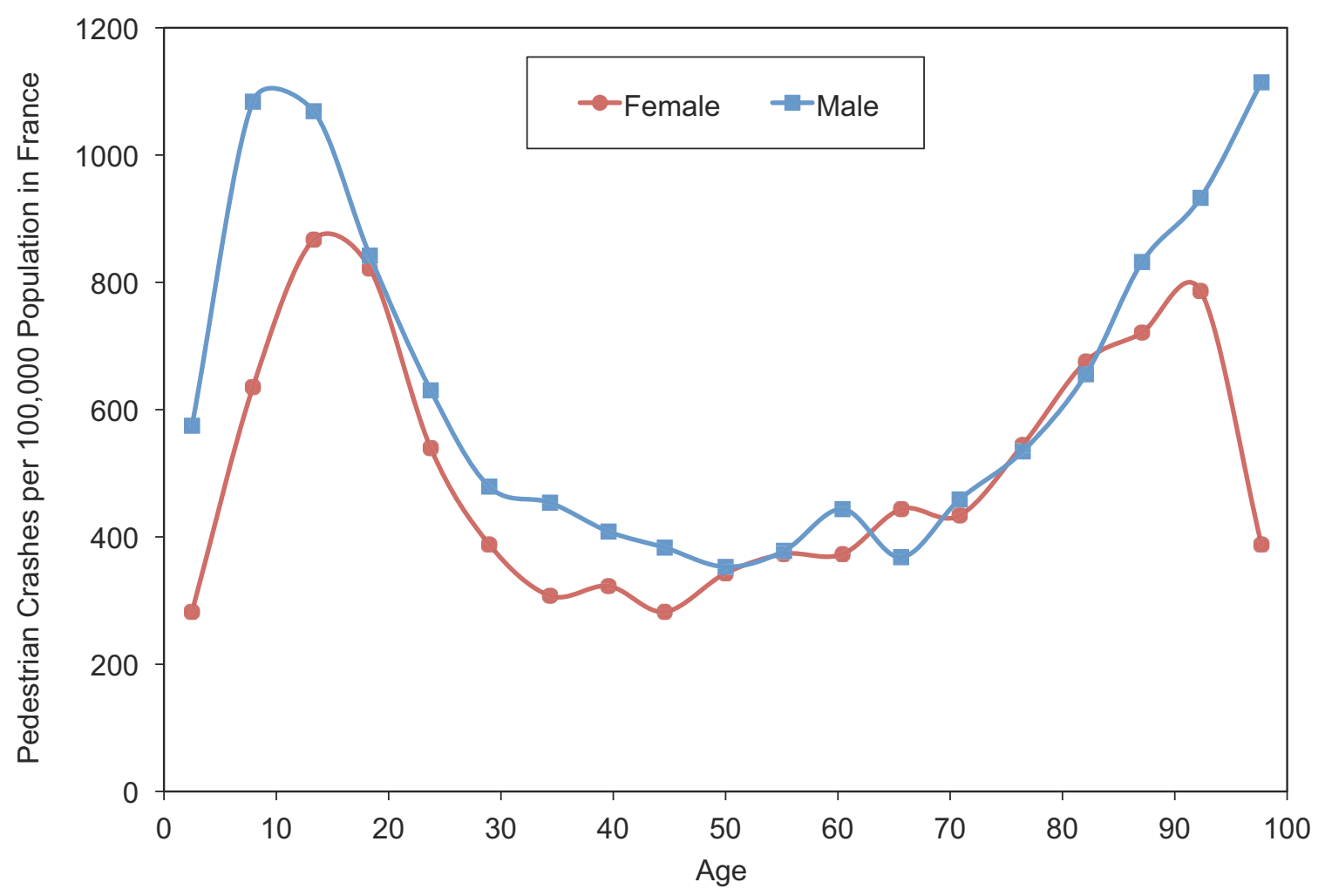

Figure 4. Incidence rates of pedestrian crashes per 100,000 population in France (Martin et al. 2011).

Even though children tend to sustain less severe injuries in pedestrian crashes than adults, their short stature produces different injury patterns than adult pedestrians, and their injuries result from different sources. For example, children are more likely to be thrown forward or knocked down in vehicle-to-pedestrian crashes. The hood is the most common source for pediatric head injuries while the windshield is the most common source of head injuries for adults. Consequently, pedestrian-friendly vehicle design must consider the difference between children and adults, addressing these two populations separately.

Figure 5 shows estimated probability of fatal-injury per crash as a function of pedestrian age (Kim et al. 2008). Age plays a significant role in determining the injury risk in pedestrian crashes, which is consistent with other types of vehicular crashes (Morris et al. 2002; Kent et al. 2005; Hu et al. 2007). Injury biomechanics literature has shown that older people are more fragile than younger adults, meaning that they tend to sustain more severe injuries for a given level of impact loading (Zhou et al. 1996; Kent et 
al. 2005; Laituri et al. 2005). At the same time, older people are also more frail than younger adults, meaning that they tend to sustain worse outcomes at a given injury ( $\mathrm{Li}$ et al. 2003; Kent et al. 2009). With the increase in aging population, many vehicle companies have considered design features specifically for older drivers and passengers (Eby and Molnar 2012), and pedestrian-friendly vehicle design should be no exception.

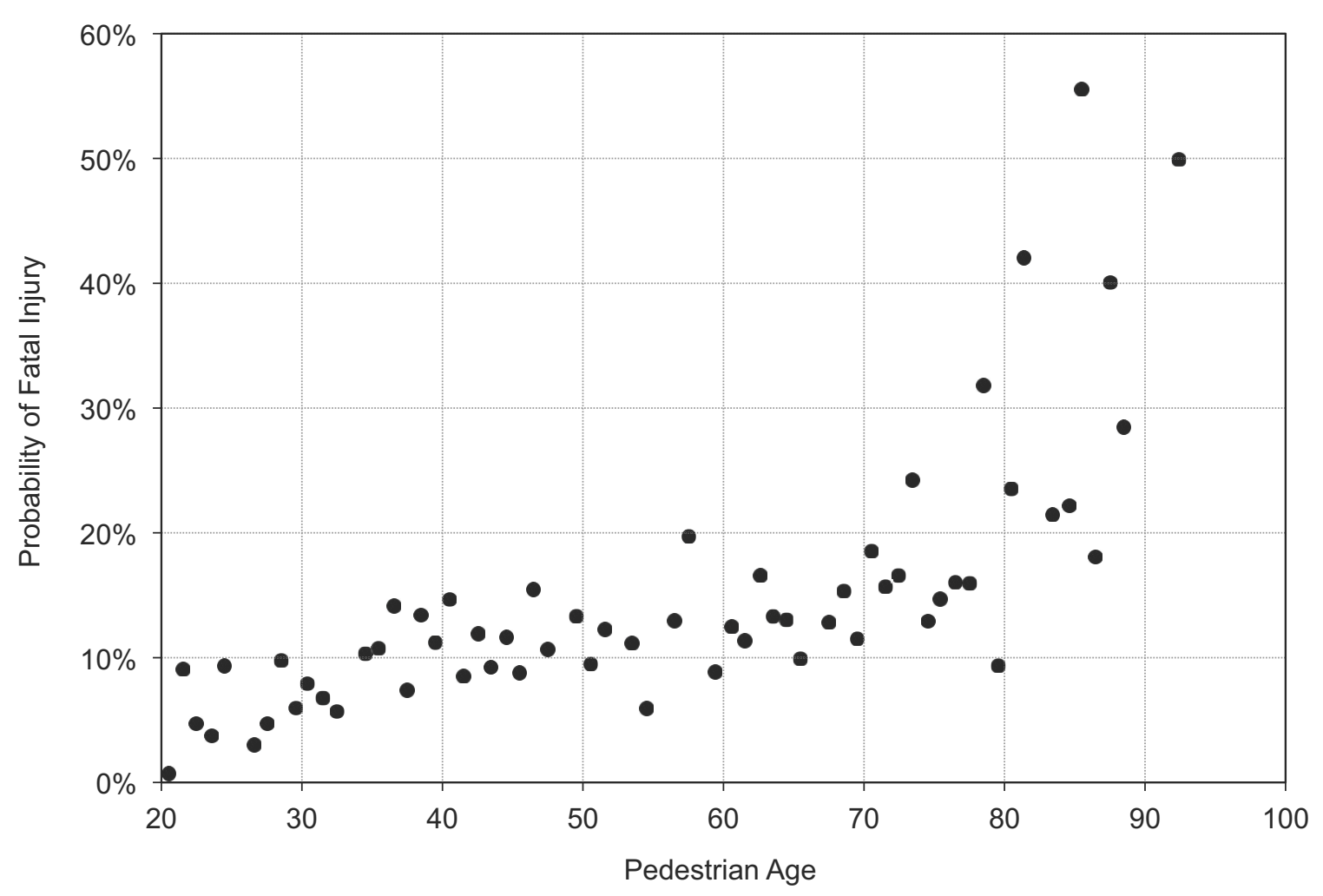

Figure 5. Fatality risk per crash as a function of pedestrian age (Kim et al. 2008).

\section{Pedestrian Injury Risk as a Function of Impact Speed}

Impact speed is the most significant crash factor affecting the injury risks in vehicle-to-pedestrian crashes. Impact speed is the measure used to describe the severity of a pedestrian crash, and is estimated through crash reconstructions using vehicle damage and pedestrian kinematics in a manner similar to how delta $\mathrm{V}$ is used to estimate vehicle crash severity using measured vehicle damage. Although the strong correlation between the impact speed and pedestrian injury risk has been well established based on pedestrian crash data (Yaksich 1964; Ashton and Mackay 1979; Pasanen and Salmivaara 
1993; Anderson et al. 1997; Davis 2001; Cuerden et al. 2007; Oh et al. 2008; Rosen and Sander 2009; Kong and Yang 2010), the absolute pedestrian injury risk as a function of impact speed is still controversial (Rosen et al. 2011). Specifically, a recent literature review (Rosen et al. 2011) found that studies conducted before 2000 were all based on direct analyses of data with oversampling of severe and fatal injuries, resulting in overestimation of pedestrian fatality risks. On the other hand, more recent studies based on less biased data provided substantially lower risk estimates than those previously reported. Figure 5 shows the fatality risk functions from different studies. Regardless of the absolute values of fatality risks, there is a consensus that pedestrian fatality risk increases monotonically with vehicle impact speed. However, the absolute risk function may affect the benefit estimation derived from using active safety features that reduce the impact speed.

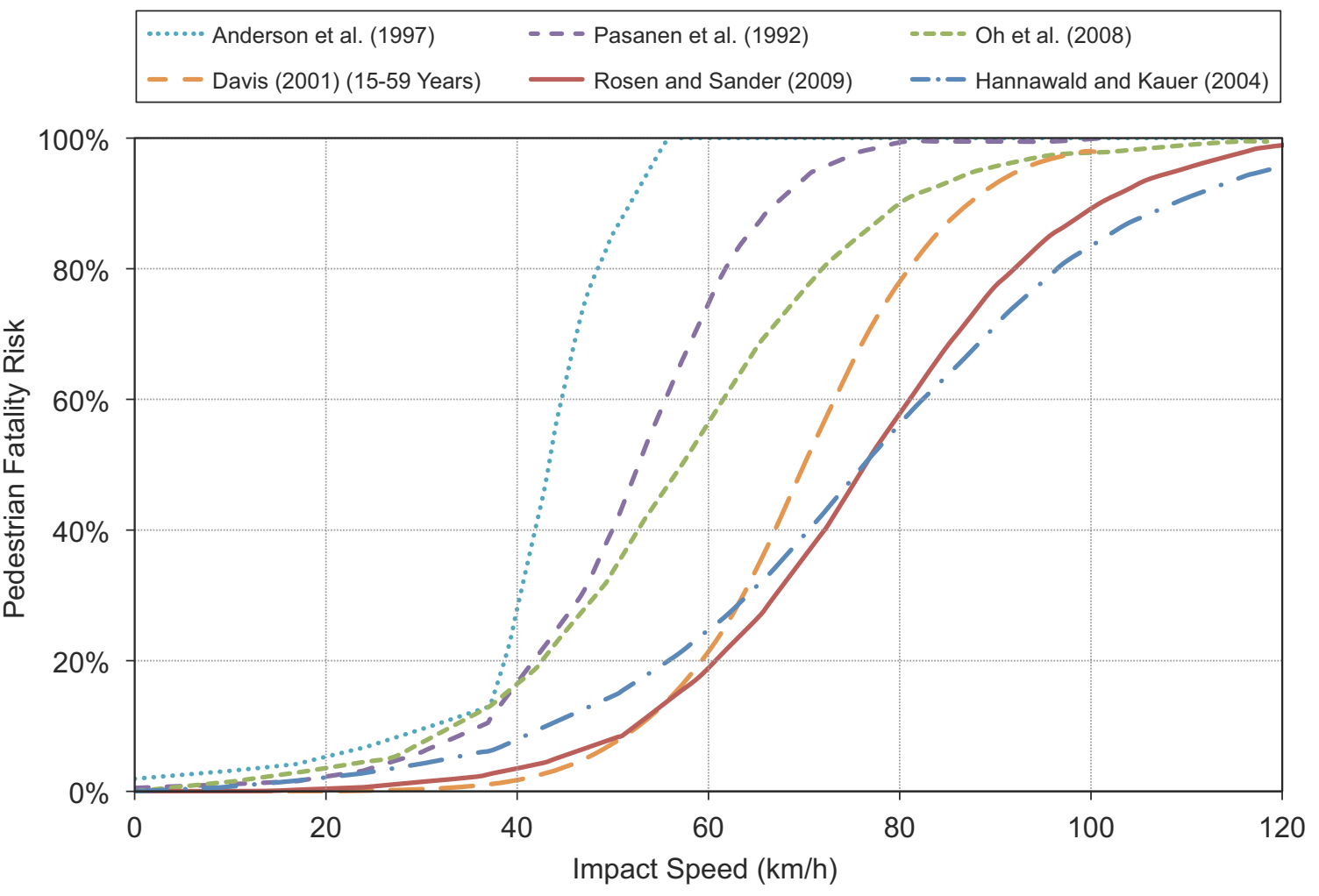

Figure 6. Fatality risk as a function of impact speed in vehicle-to-pedestrian crashes (Rosen et al. 2011). 


\section{Vehicle Type Effects on Pedestrian Injuries}

Apart from safety design features for a pedestrian-friendly vehicle, many recent studies have shown that vehicle type has a strong effect on pedestrian-injury and fatality risk (Lefler and Gabler 2004; Roudsari et al. 2004; Longhitano et al. 2005; Paulozzi 2005; Desapriya et al. 2010). LTVs, (pickup trucks, vans, and SUVs), were reported to be associated with three times higher risk of severe pedestrian injuries and more than three times higher fatality risk than cars (Roudsari et al. 2004). Furthermore, LTVs result in different pedestrian-injury patterns than those from cars. In particular, although the head is the most commonly injured body region for both LTVs and cars, the lowerextremity region is the second for cars, whereas the torso is the second for LTVs. The most frequent injury sources for cars are the windshield and the bumper, while it is the hood and hood leading edge for LTVs. More recently, Desapriya et al. (2010) performed a literature review to quantify the vehicle type on fatal pedestrian injuries based on 11 previous studies. The overall pooled data led to an odds ratio of 1.54 for fatal pedestrian injuries with LTVs compared with cars.

Figures 7 and 8 provide a more detailed view of the injury-pattern and injurysource differences between LTVs and cars. Overall, LTVs cause more injuries throughout the whole body of a pedestrian than cars, but the torso region showed disproportionally higher injury risk associated with the hood and hood edge in LTVs.

Compared with cars, LTVs are generally heavier, stiffer, higher, and geometrically more blunt. However, because of the mass disparity between a vehicle and a pedestrian, the mass difference between LTVs and passenger vehicles does not pose significant effects on the injury risk. Thus, the stiffness, higher center of gravity, and geometry of the LTVs' front-end design are likely the major reasons for the difference in pedestrian-injury risk. 


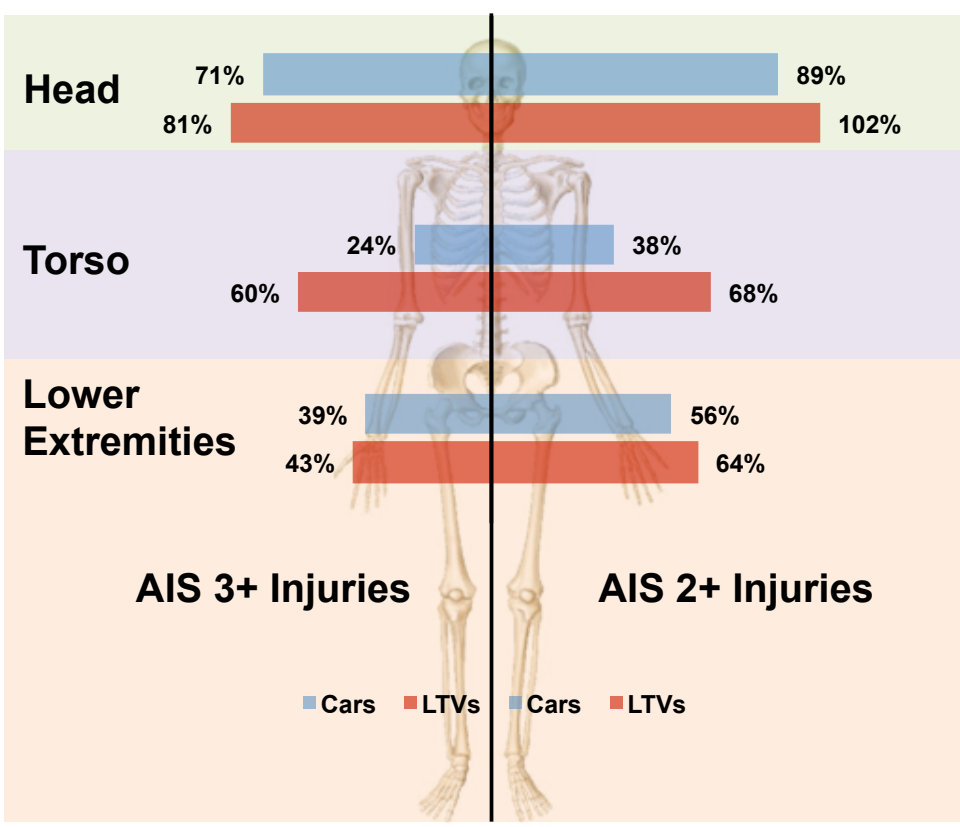

Figure 7. Distribution of pedestrian injuries by vehicle type (Longhitano et al. 2005). 

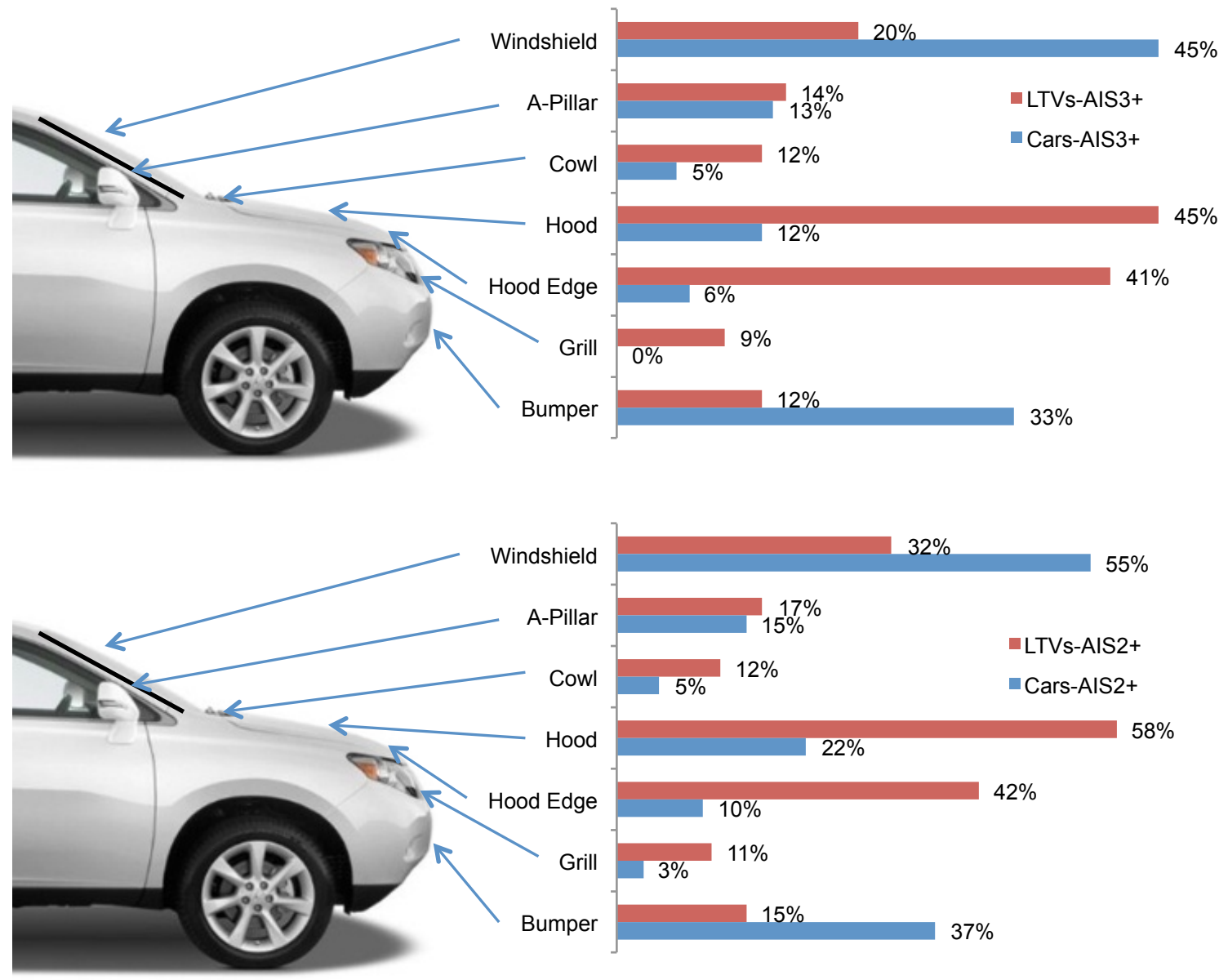

Figure 8. Sources of pedestrian injuries by vehicle type (Longhitano et al. 2005).

\section{SUVs and Older Pedestrians}

Age and vehicle type are two important factors affecting the injury risks in vehicle-to-pedestrian crashes. Interestingly, there are currently two independent trends in the world, especially in developed countries, with one being the aging of the population (Figure 9), and the other the increasing proportion of SUVs (Figure 10). Unfortunately, both of these trends tend to increase the pedestrian-injury risk. Consequently, addressing the hazards posed by SUVs to older pedestrians is an important traffic-safety challenge (Desapriya and Pike 2005; Simms and O'Neill 2005; Watts 2005). Better understanding of the decreasing injury tolerance of the older population, and of the injury patterns, sources, and mechanisms associated with SUV-to-pedestrian crashes will be important for addressing optimal vehicle designs. 


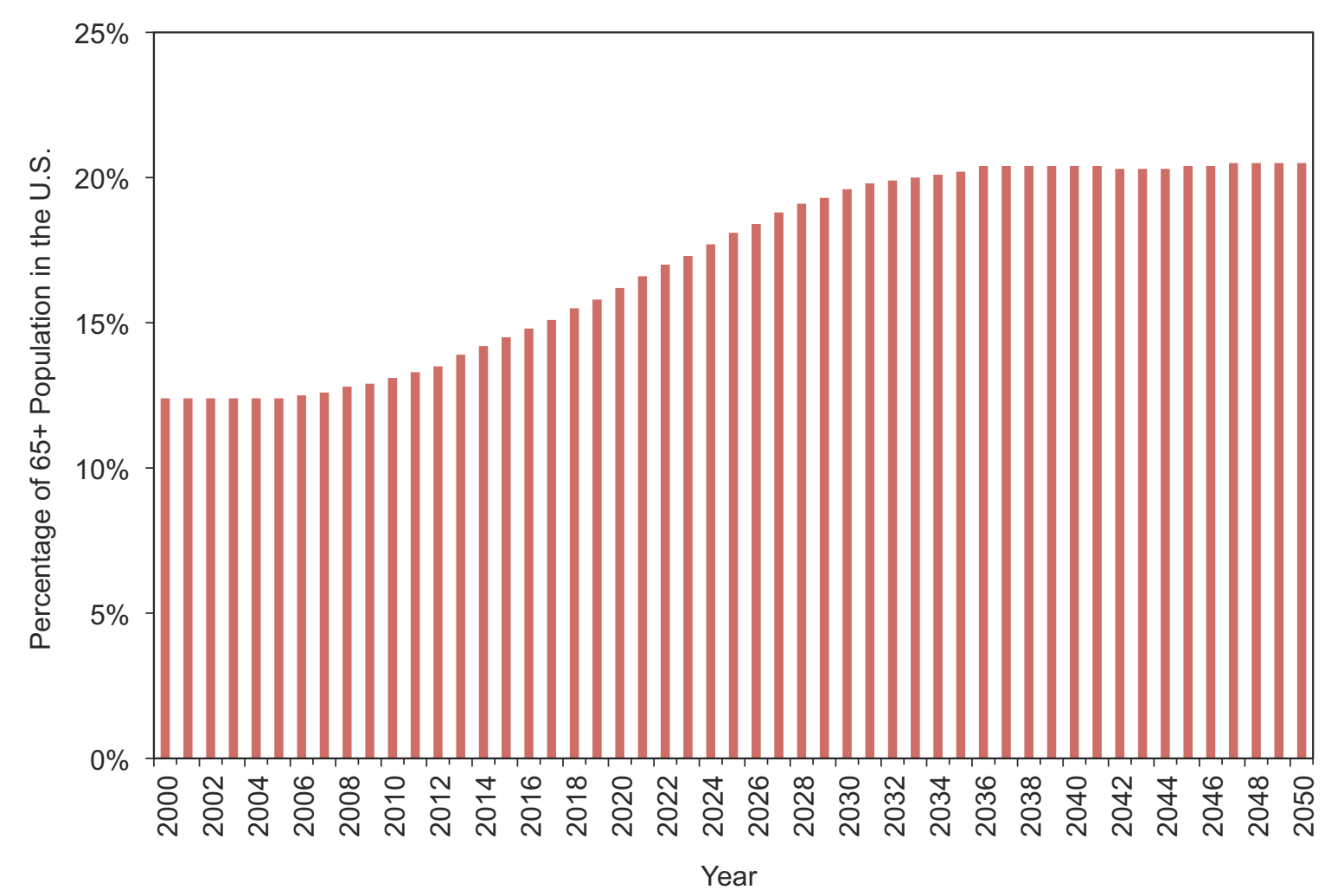

Figure 9. Percentage of $65+$ year-old population in the U.S. (http://www.census.gov). 


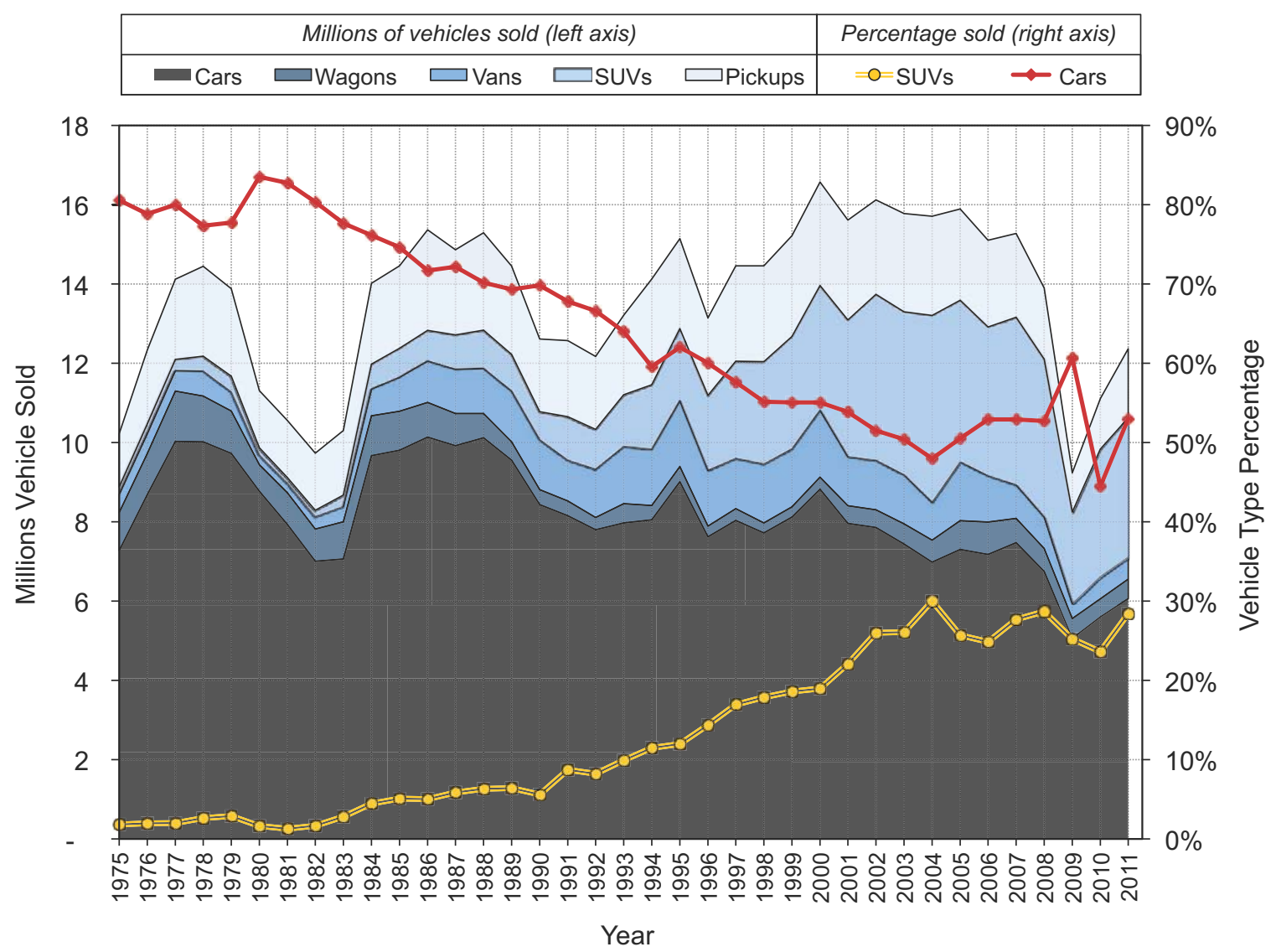

Figure 10. Light vehicles sold in the U.S. (http://www.afdc.energy.gov). 


\section{Test Procedures for Pedestrian Protection}

In spite of the globally recognized safety concerns regarding pedestrian injuries, no safety standards currently exist for pedestrian protection in the U.S. In contrast, Europe, Japan, and Australia consumer test programs and regulations include testing procedures for evaluating the pedestrian protection of vehicle designs. These include the New Car Assessment Program (NCAP), pedestrian impact tests in a European Directive (2003), and the pedestrian headform test in Japan (Nishimoto 2003). However, because of the model overlap among different markets, the pedestrian protection of the vehicles in the U.S. is inevitably influenced by the test procedures in other countries, especially those in Europe.

Pedestrian safety tests have been proposed by a variety of different organizations, including the working groups of the European Enhanced Vehicle Safety Committee (EEVC), the International Organization for Standardization (ISO), and the IHRA. However, the test procedures are all very similar. All of these tests are designed to replicate vehicle-to-pedestrian crashes at $40 \mathrm{~km} / \mathrm{h}$, and use individual component tests representing impacts to different body regions instead of full-scale dummy tests. An impact speed of $40 \mathrm{~km} / \mathrm{h}$ is used, because it is believed to be a reasonable upper speed that covers most pedestrian crashes and injuries (Mizuno 2005). The isolated component tests are used because full dummy tests are not as repeatable, especially for simulating secondary head impacts. The goal of the isolated component tests is to reproduce the impact between a pedestrian body part and its corresponding vehicle part, and assess the injury risk based on the injury-risk curves developed from cadaver tests. Due to the similarity of different pedestrian test procedures, this report discusses only the Euro NCAP procedure for pedestrian protection, which follows the EEVC guidelines.

\section{Euro NCAP Pedestrian Protection Test Procedure}

Based on pedestrian injury data, the head and lower extremities are the most commonly injured body regions in vehicle-to-pedestrian crashes. As a result, Euro NCAP (and all the other pedestrian impact-test procedures) focuses only on these two body regions. As shown in Figure 11, Euro NCAP pedestrian tests include legform to bumper tests, upper legform to hood leading-edge tests, and child/adult headform to 
hood/windshield tests. Multiple tests are conducted at different test zones at the bumper, hood leading edge, and headform contact areas. The headform test area is defined based on the pedestrian wrap-around distance (WAD) as shown in Figure 12, in which child and adult headform test zones are separated. The test zones cover almost the full width of the vehicle, so that the overall pedestrian protection can be evaluated throughout the vehicle front-end structures. Once the tests are conducted, the impact responses are then assessed by points and rated in color. The impact responses measured in the pedestrian impact tests and their associated injury criteria are shown in Table 4. Maximums of 6, 6, and 24 points are available for the bumper, hood leading edge, and headform test zone, respectively, leading to a total of 36 points available in the pedestrian protection assessment. Before 2009, Euro NCAP released a separate star rating for pedestrian protection; starting in 2009 the above score system has become an integral part of the overall rating scheme.
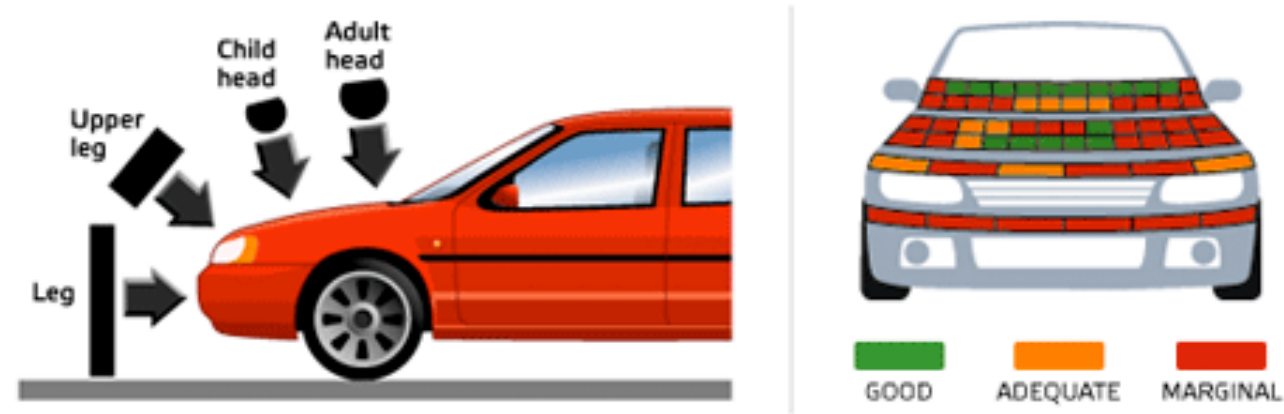

Figure 11. Euro NCAP pedestrian protection test and rating (Euro NCAP 2012a; Euro NCAP 2012b). 

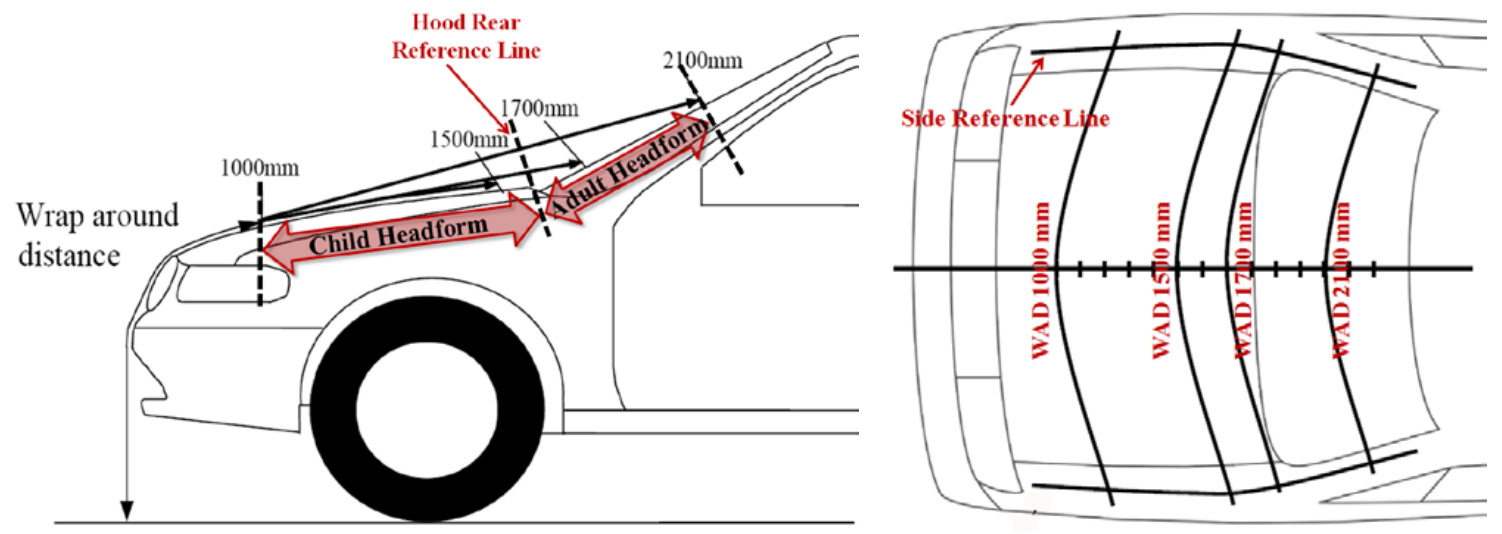

Figure 12. Headform test zones based on wrap around distance (Euro NCAP 2012b).

Table 4

Injury criteria in Euro-NCAP pedestrian impact tests

(EuroNCAP 2012; EuroNCAP 2012).

\begin{tabular}{|c|c|c|c|c|c|c|}
\hline $\begin{array}{l}\text { Body } \\
\text { Region }\end{array}$ & Injury Measurements & \multicolumn{3}{|c|}{$\begin{array}{l}\text { Higher limit } \\
\text { (1.0 point) }\end{array}$} & \multicolumn{2}{|c|}{$\begin{array}{l}\text { Lower limit } \\
\text { (0.0 point) }\end{array}$} \\
\hline \multirow{3}{*}{ Legform } & Tibia deceleration & \multicolumn{3}{|c|}{$150 \mathrm{~g}$} & \multicolumn{2}{|l|}{$200 \mathrm{~g}$} \\
\hline & Knee shear displacement & \multicolumn{3}{|c|}{$6 \mathrm{~mm}$} & \multicolumn{2}{|l|}{$7 \mathrm{~mm}$} \\
\hline & Knee bending angle & \multicolumn{3}{|c|}{$15^{\circ}$} & \multicolumn{2}{|l|}{$20^{\circ}$} \\
\hline \multirow{2}{*}{$\begin{array}{l}\text { Upper } \\
\text { Legform }\end{array}$} & Bending moment & \multicolumn{3}{|c|}{$300 \mathrm{Nm}$} & \multicolumn{2}{|l|}{$380 \mathrm{Nm}$} \\
\hline & Sum of forces & \multicolumn{2}{|r|}{$5.0 \mathrm{kN}$} & \multicolumn{3}{|c|}{$6.0 \mathrm{kN}$} \\
\hline \multirow{3}{*}{ Headform } & \multirow{3}{*}{$\begin{array}{l}\mathrm{HIC}_{15} \\
\text { (Head Injury Criterion) }\end{array}$} & $<650$ & $650 \sim 1000$ & $1000 \sim 1350$ & $1350 \sim 1700$ & $>=1700$ \\
\hline & & Green & Yellow & Orange & Brown & Red \\
\hline & & $\begin{array}{l}1.00 \\
\text { point }\end{array}$ & 0.75 point & 0.5 point & 0.25 point & $\begin{array}{l}0.00 \\
\text { point }\end{array}$ \\
\hline
\end{tabular}

\section{Limitations of Current Pedestrian Impact-Testing Procedures}

The EEVC, ISO, IHRA, and Euro NCAP pedestrian testing systems are all comprehensive programs for pedestrian protection, which are designed according to the injury patterns in the field and injury tolerance in cadaver tests. However, these testing procedures have several limitations.

First, the current pedestrian impact tests use isolated component tests, which mainly focus on the stiffness and energy absorbing capability of the isolated vehicle 
front-end structures. The overall shape, the transitions between those structures, and the vehicle-type-related geometry differences cannot be fully evaluated.

Second, according to the pedestrian injury data, torso injuries are the second most common injuries to cause fatality, but they have not been considered by the current pedestrian impact tests. Given the fact that SUVs cause more torso injuries than cars, and elderly are more likely to suffer from chest injuries, the increased proportions of SUVs among the vehicle fleet and the increased proportion of older people among the population will likely increase the importance of pedestrian torso protection.

Finally, the current pedestrian tests only evaluate passive safety designs, which are indeed essential for pedestrian protection. However, with the fast development of active safety features, future pedestrian standards will inevitably require testing procedures for evaluating the effectiveness of active safety designs as well. Such procedures are not yet available.

Despite these limitations, the current testing procedures for pedestrian protection have resulted in significant pedestrian-friendly changes in vehicle design. Further introduction of full-body dummy tests and computational human modeling will provide even more comprehensive evaluation of pedestrian-protection capability. 


\section{Vehicle Designs for Pedestrian Protection}

\section{Basic Design Theories of Pedestrian-Friendly Vehicles}

From an injury-biomechanics point of view, reducing the injury risk in a vehicleto-pedestrian crash can be achieved in two ways: reducing the impact energy or prolonging the duration of each impact. The former is achieved by reducing the impact speed, and the latter by reducing the contact stiffness and increasing the crush depth. However, in most real vehicle-to-pedestrian crashes, a specific sequence of impacts occurs, starting from the bumper-to-leg impact, followed by the pelvis/abdomen/chest-tohood-leading-edge impact, and ending with hood/windshield-to-head impact. As a result, the velocity of a latter impact (such as to the head) is associated with the outcome of the previous ones (such as an impact to the leg). Therefore, the vehicle shape (or geometry) also has profound influence on the overall pedestrian injury risk, especially on the mid torso and head, which are generally caused by secondary impacts. Furthermore, due to the significant variations in vehicle geometry and pedestrian stature, the precise contact stiffness depends greatly on the exact impact location determined by the relative height and position between a pedestrian and a vehicle. Therefore, reducing the overall stiffness of the vehicle front-end structures and maintaining energy-absorbing capability to avoid "bottoming out" in a vehicle-to-pedestrian impact are essential for pedestrian protection. As mentioned earlier, although vehicle mass significantly affects occupant safety in vehicle-to-vehicle crashes, it does not greatly influence the pedestrian injury risk because of the large disparity in mass between a pedestrian and a moving vehicle (Lefler and Gabler 2004; Simms and Wood 2006).

In the past four decades, many studies have been performed with the focus of optimizing the shape and stiffness of the vehicle front-end structures for pedestrian protection. The development of impact-sensing systems enabled passive, deployable safety designs for protecting pedestrians that include pop-up hoods and windshield airbags. More recently, active safety features, such as braking-assist and autonomousbraking systems were proposed to avoid collisions or reduce the vehicle travelling speed before the impact with a pedestrian. The integration of pedestrian safety into the NCAP system has motivated vehicle manufacturers to produce pedestrian-friendly vehicle designs for reaching a better NCAP rating. This trend will likely lead to a much better 
overall pedestrian-friendly stiffness distribution in the vehicle front-end structures, but active safety systems have not yet been incorporated into the evaluation system.

\section{Designs for Reducing Pedestrian Lower-Extremity Injuries}

Because pedestrian lower-extremity injuries are primarily caused by direct loading from the bumper, most vehicle designs for reducing pedestrian lower-extremity injuries have focused on the bumper. While the hood leading edge also affects injury to the upper portion of the lower extremities in pedestrian impacts, it is more difficult to modify its geometry and stiffness to meet relatively stringent requirements in pedestrian test programs. However, considering the bumper and hood leading edge as a whole is important for pedestrian protection purposes.

\section{Bumper Designs}

Bumper designs vary in height, depth, and stiffness. The bumper height is generally predetermined by vehicle type, the bumper depth is influenced by the styling and the goal of a smooth transition from the bumper to the hood leading edge, and the bumper stiffness is constrained by its original function of protecting the vehicle in lowspeed impacts.

The bumper-height effects on the type of pedestrian lower-extremity injuries have been discussed by Matsui (2005). With an increased bumper height, the tibia/fibula fractures and knee injuries could transition to injuries to the femur mainly due to the higher impact location. Interestingly, the main injuries at an impact velocity of around 40 $\mathrm{km} / \mathrm{h}$ are bone fractures, while impacts at velocities around 20 to $30 \mathrm{~km} / \mathrm{h}$ tend to injure knee ligaments. The bumper height alone does not necessarily affect the severity of lower-extremity injuries, but the size of the contact area likely affects the bending moment in the knee area.

From an energy-absorption point of view, the bumper depth is critical for determining the maximal energy that can be absorbed by the bumper system. However, modern vehicle styling tends to minimize the bumper protrusion in front of the vehicle in contrast to the protruded bumpers of 30 to 40 years ago (as shown in Figure 13), thus adding constraints to the bumper depth. However, previous studies have shown that a relatively smooth front-end structure increases the contact area during a bumper-to-leg 
impact, thus reducing the overall risk of lower-extremity injuries (Otte and Haasper 2005).

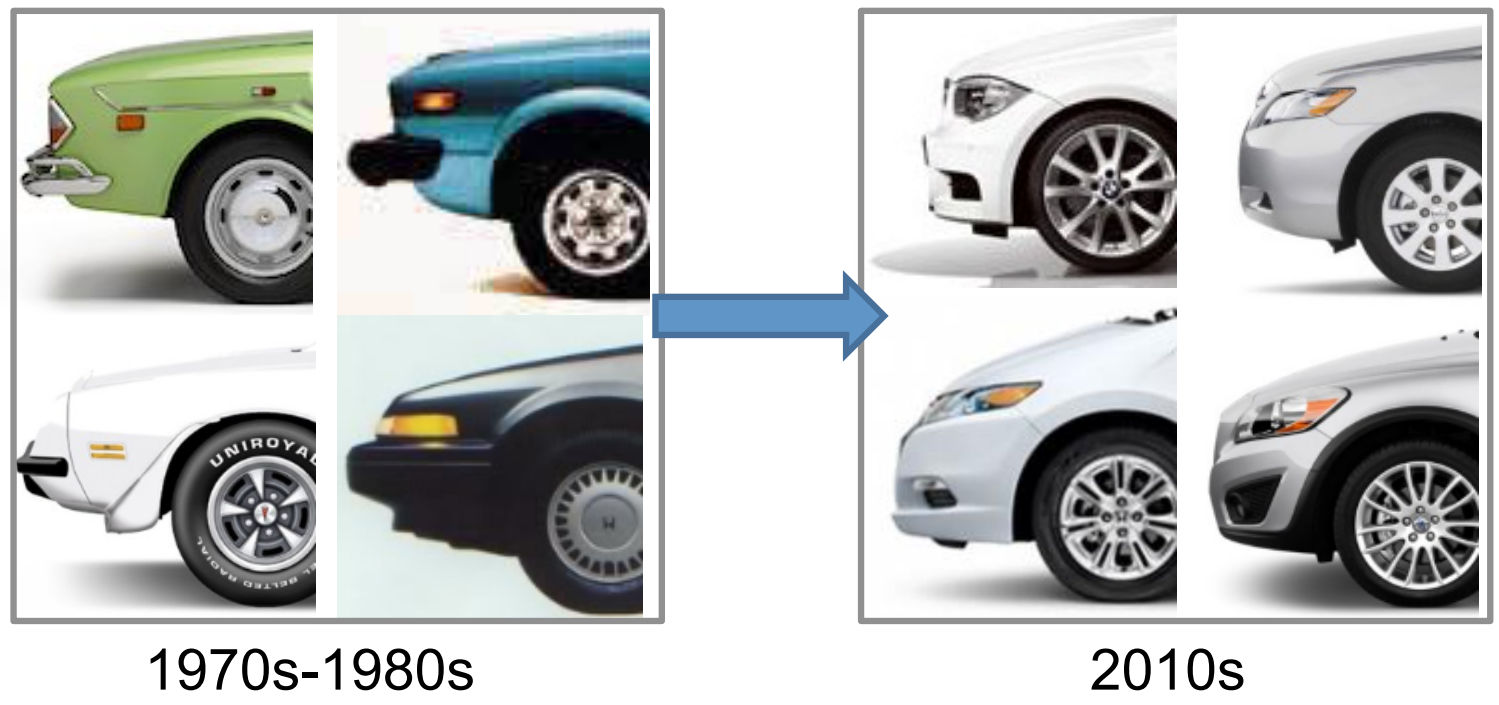

Figure 13. Change in vehicle bumper style.

The bumper stiffness is also extremely critical for pedestrian lower-extremity protection. However, the bumper's primary function is to protect the vehicle in lowspeed impacts, whose energy is much higher than that in pedestrian crashes. Consequently, the stiffness of the bumper must be high enough to absorb sufficient energy in vehicle-to-vehicle crashes, but also low enough to reduce the leg-form accelerations to avoid fractures in pedestrians. These conflicting demands limit an ideal protection for pedestrian lower-extremities, but drive the bumper designs to be more efficient in energy absorption. System optimization is necessary for this kind of multiobjective problem.

Based on the lower-extremity injury patterns shown in field data analyses (Takahashi et al. 2000; Klinich and Schneider 2003; Yang 2005) and injury mechanisms shown in cadaver tests (Kajzer et al. 1997; Kajzer et al. 1999), pedestrian lower-extremity injuries can be categorized into two types: bone fractures and knee injuries. The tibia, fibula, and femur shaft fractures are generally caused by the direct loading, predicted by the high acceleration in the legform tests, which can be reduced by cushioning the impact 
in a more efficient manner. The knee injuries, including hard and soft tissue injuries around the knee, are highly related to the bending and shear in the knee area, which is not sensitive to the stiffness of the bumper, but to the loading location and the total height of the bumper (Matsui 2005). Consequently, two types of bumper design features are popular for reducing pedestrian lower-extremity injuries. The first design feature uses foam, molded plastic, or other energy absorbers to improve the energy absorption efficiency, and the second provides additional support of the lower leg (such as fixed/deploying lower stiffeners and bumper airbag) to reduce the knee bending (Schuster 2006).

In today's vehicles, the bumper system generally consists of a plastic bumper face over an energy absorbing material supported by immovable structures. The stiffness and size of the energy absorbing material can be optimized to meet the pedestrian legform test and vehicle-to-vehicle low-speed test at the same time. For example, approaches have used a combination of deforming loop and crush cans to provide a two-stage energy management in its bumper system, plus a deformable plastic box behind the bumper fascia and an energy-absorbing under-tray to support the spoiler (Hardy et al. 2006).

Pedestrian-friendly bumper designs have to consider the full width of the vehicle and full length of the legform. A study by Matsui et al. (2011) found that foam materials in the bumper system had significant effects on reducing the tibia fracture risk against vehicle bumper center impacts, but had little effect at the sides of the bumper due to the thinner foam materials. The front shape and the stiffness discrepancy along the length of the legform also affected the bending angle of the legform.

Because the legform is generally longer than the height of the bumper, a concentrated loading would very likely cause bending of the knee, even if the loading alone is relatively small. A vertically larger bumper can significantly reduce this type of bending, which can be achieved by adding a lower stiffener or bumper airbag. The protection effects provided by lower stiffeners and bumper airbags have been demonstrated by computational simulations and tests (Schuster and Staines 1998; Pipkorn et al. 2007). However, it is challenging to use these types of devices in vehicles, because the fixed lower stiffener will reduce the vehicle bumper clearance and affect vehicle 
styling. Furthermore, deployable stiffeners and bumper airbags are relatively expensive and must rely on a robust sensing system to detect the collision beforehand.

\section{The Hood Leading-Edge Designs}

The effects of hood leading edge on pedestrian lower-extremity injuries are understood rather well. Recent studies using a computational human model (Snedeker et al. 2003) and a pedestrian crash dummy (Snedeker et al. 2005) have shown that a low hood leading-edge height $(<750 \mathrm{~mm})$, a large hood-edge radius $(>250 \mathrm{~mm})$, a moderate bumper lead (>150 $\mathrm{mm})$, and a sufficiently high bumper-edge height $(>490 \mathrm{~mm})$ would virtually eliminate pelvis and femur fractures in a collision with a 50th percentile male pedestrian at speeds less than $40 \mathrm{~km} / \mathrm{h}$. The roundness of the hood leading edge is even more critical in vehicles with a moderate leading-edge height $(750-850 \mathrm{~mm})$, while a leading-edge height over $850 \mathrm{~mm}$ may be over the hip, resulting in contact with the torso. The contact speed between the pelvis/thigh and the hood also largely depends on the roundness of the hood leading edge, which is not considered by the isolated component tests for pedestrian protection.

Aside from the geometries of the hood leading edge, its stiffness is also critical for pedestrian protection. Unfortunately, most current hood leading-edge designs are too stiff, because they are generally supported by relatively rigid structures, including the hood latch, the housing of the headlamps (Hardy et al. 2006), and the reinforced edge. However, studies have shown that the hood latch can be designed to allow downward movement (Kalliske and Friesen 2001), the lamp housing can be made deformable to reduce the hood leading-edge stiffness, and the hood front edge can be moved rearward (Hardy et al. 2006).

In spite of improvements to the hood leading edge for pedestrian protection, concerns were posed by car manufactures regarding the upper-legform tests (Hardy et al. 2006). In particular, the upper-legform test requirements were considered to be too stringent. Consequently, car manufacturers tended to put more effort into improving the bumper and the hood than the hood leading edge. Further investigations are necessary to reevaluate the upper-legform test procedure and requirements. Research in the 1980s included a focus on preventing pedestrian torso injuries, because pedestrian torso injuries from passenger cars was a bigger problem before vehicle profiles changed from more 
rectangular to more sloped designs in the 1980s. Some of this earlier research may be worth revisiting because the front profiles of SUVs somewhat resemble the front profiles of 1970s passenger vehicles.

\section{Designs for Reducing Pedestrian Head Injuries}

Analogous to the design theories for reducing pedestrian lower-extremity injuries, reducing pedestrian head injuries also requires vehicle designs with lower impact stiffness and greater crush zone. Given the fact that most pedestrian head injuries are caused by the hood and windshield, the energy absorbing capability of these two components are of main interest for pedestrian head protection. Because the hood is made of sheet metal and the windshield is made of laminated glass, both of them are relatively compliant structures and do not, by themselves, pose major risk for severe head injuries (Crandall et al. 2002). However, serious head injuries could occur if the head hits a region of the hood with underlying rigid engine components. Moreover, the hood is generally reinforced on the edge and the windshield is supported by the stiff A-pillars and cowl. Field data have shown that fatal injuries are more common from impacting the side of the hood and windshield frame than their center areas, which are now generally designed to have a gap between the hood and underling components. Because pedestrians vary significantly in height, the exact impact location may also vary significantly. Therefore, to achieve a better pedestrian head protection, hood design must focus on optimizing the energy-absorbing capability throughout the area (including the edge) and at the same time leaving enough crush space to avoid "bottoming out." On the

other hand, it is difficult to reduce the stiffness of the windshield frame, because those frames also serve as important support structures to ensure the integrity of the occupant compartment. Therefore, deployable countermeasures, such as a windshield airbag, may be necessary to ensure the proper energy absorption in those areas.

\section{Hood and Pop-up Hood Designs}

Numerous studies have demonstrated the effectiveness of redesigning the hood structure to achieve a more uniform stiffness profile and better energy-absorbing efficiency. For example, Kerkeling et al. (2005) conducted pedestrian impact tests on various hood concepts with different hood and hood-hinge designs as shown in Figure 
14a-c. Pedestrian head protection can be improved by introducing more energyabsorbing hood designs with collapsible multijoint hinges. Following the same concept, Liu et al. (2009) proposed a sandwich hood structure (Figure 14d) with potential for improving pedestrian head protection with a relatively small underhood clearance (60 $\mathrm{mm}$ for child head and $75 \mathrm{~mm}$ for adult head). Belingardi et al. (2009) presented a hybrid hood concept, in which a thermoplastic or wire inner structure and an outside metal panel were combined to achieve better energy absorption (Figure 14e-f).

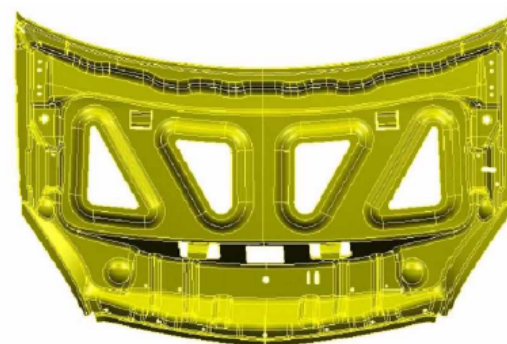

(a) Traditional Design

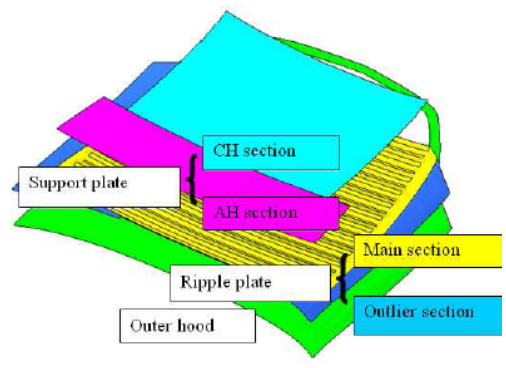

(d) Sandwich Design

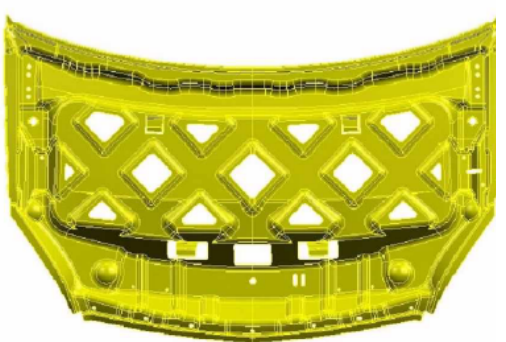

(b) Increased Rib Design

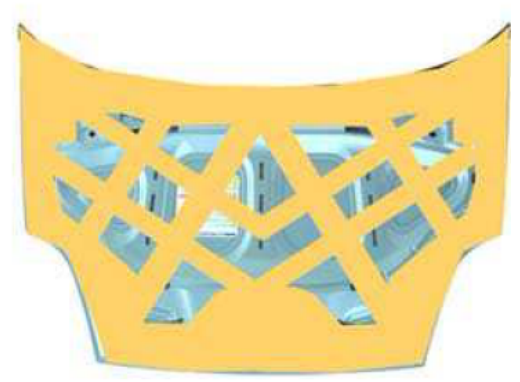

(e) Hybrid Design Concept

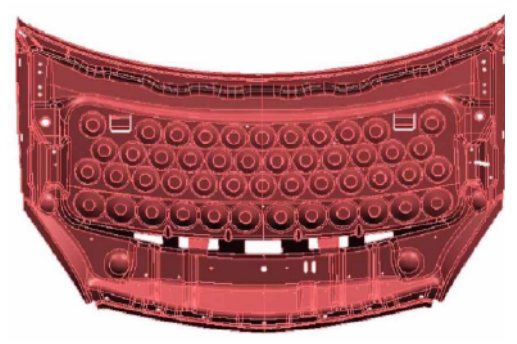

(c) Multi Cone Design

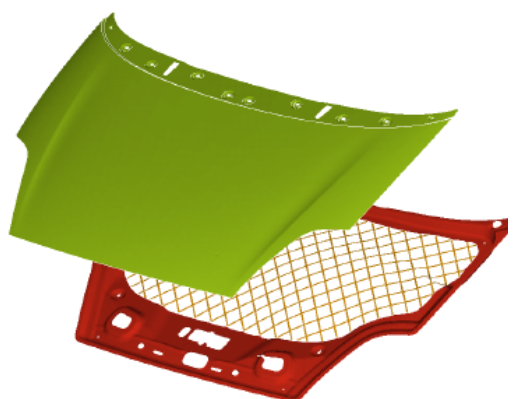

(f) Wire Hybrid Concept

Figure 14. Different hood designs for improving pedestrian head protection (a)-(c) are from Kerkeling et al. (2005), (d) is from Liu et al. (2009), and (e) and (f) are from Belingardi et al. (2009).

Despite new hood-structure designs and their increased energy-absorbing efficiency, bottoming out during a pedestrian head impact cannot be easily avoided with limited underhood clearance. This is especially true for sports cars, in which the hood needs to be lower to achieve better aerodynamics, styling, and visibility. A pop-up hood is a design that can solve this problem (Figure 15a). Several previous studies have presented designs and evaluation of the effectiveness of a pop-up hood system (Fredriksson et al. 2001; Lee et al. 2007; Oh et al. 2008; Shin et al. 2008; Inomata et al. 
2009; Huang and Yang 2010; Evrard 2011). Such a system provides additional space between the hood and the rigid components beneath by raising the rear of the hood before a pedestrian head impact. As a result, larger hood deformation and more impact energy can be absorbed without bottoming out, and thus the head injury risk can be reduced in pedestrian crashes. Because it is a deployable design, it is a complex system, generally including a bumper sensor, an electronic control unit (ECU), a hood actuator, and a hoodhinge release mechanism.

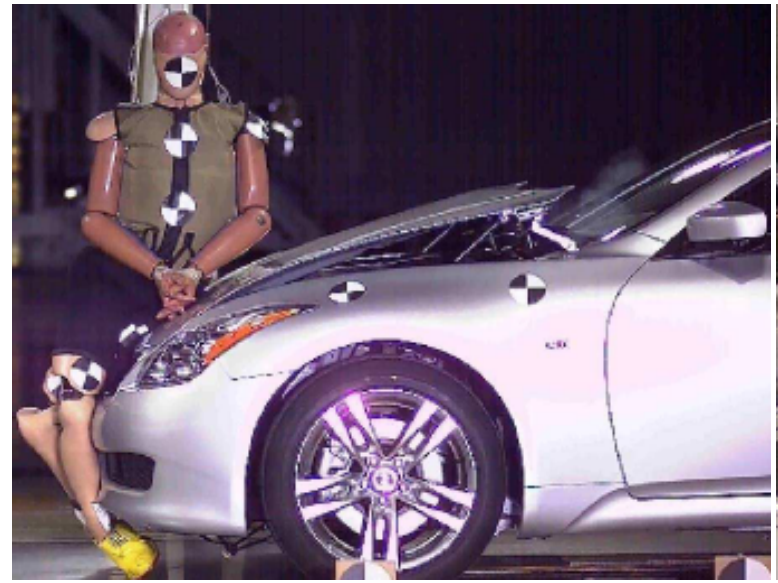

Pop-up hood (Inomata et al. 2009)

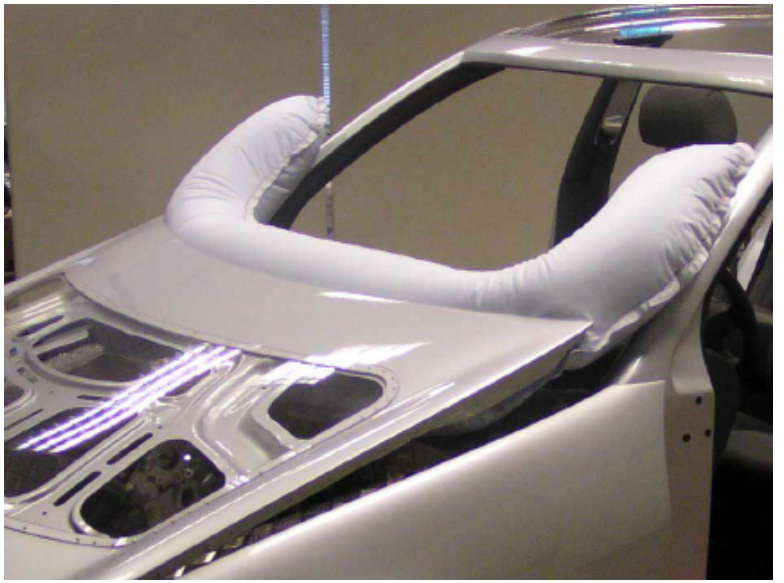

Windshield airbag (Kuehn et al. 2005)

Figure 15. Deployable passive safety devices for pedestrian protection.

\section{Windshield and Windshield Airbag}

Pedestrian injury data have shown that windshields account for over $50 \%$ of pedestrian head injuries. The windshield itself is generally made of laminated glass, which is fairly compliant during crashes. However, the particular type of laminated glass employed has been found to be a significant factor affecting the head injury risk in pedestrian testing (Pinecki et al. 2011). Specifically, Xu et al. (2011) found that the nanoporous material can absorb more energy than the conventional interlayer material of laminated glass. Obviously, due to the multilayer nature of laminated glass, more energy absorbing material is available for further improvement of pedestrian head protection.

In addition to the effects of the windshield material, the impact point close to the windshield frame strongly increases the head injury risk. It has been reported that a 
distance of $110 \mathrm{~mm}$ from the windshield frame is needed to achieve a HIC (head injury criterion) of less than 1000 (Pinecki et al. 2011). Due to the difficulty of reducing the stiffness of the A-pillars and cowl, a windshield airbag (Figure 15b) provides probably the best solution for solving this problem. The windshield airbag can also serve as an additional protection device with the pop-up hood, so that the gap between the popped hood and the windshield frame can be filled. However, similar to the pop-up hood, a relatively complex system is needed for designing a reliable windshield airbag.

\section{Active Safety Designs}

Active safety designs were not considered much until the last few years. Recent rapid development of sensing systems for detecting an imminent collision enabled active safety features to be used for occupant protection, and similar technology can also be used for pedestrian protection. The goal of all active safety designs for pedestrian protection is to avoid a pedestrian crash or reduce the impact speed. Given the strong correlation between the impact speed and pedestrian injury risk, such technology, if reliable, would significantly reduce all pedestrian injuries regardless of the specific body region.

Although active safety designs for pedestrian protection are all involved in some types of pre-impact braking, the specific technologies to detect an imminent pedestrian crash can be different. For example, current Brake Assist Systems (BASs) were developed for improving drivers' braking performance in emergency situations. The theory behind these systems is that during emergency braking most drivers do not make effective use of the full capability of the braking system (Perron et al. 2001). Therefore, a BAS will be triggered to maximize the braking when a driver intends to make an emergency stop. The algorithm for detecting the emergency situation can vary, but is generally associated with the brake pedal speed, force, or brake fluid pressure. Because current BASs have to rely on drivers' braking action, it would be expected to activate in only about $50 \%$ of all crashes (Hannawald and Kauer 2004). To enhance the performance of a BAS, forward looking sensors can be incorporated. Such sensors can offer a wide range of possibilities, such as driver warning, BAS enhancing, or autonomous braking (Hardy et al. 2006). The sensors can vary from radar sensors to 
vision sensors. Stereo cameras, as one type of vision sensors, have been used for detecting impending pedestrian crashes (Matsui et al. 2011).

Considerable evidence suggests that active safety designs could benefit pedestrian protection. However, the reliability and effectiveness of such designs are still uncertain. Interestingly, if the reliability can be ensured in the active sensing system, early detection of a pedestrian crash can provide more possibility of implementing additional passive deployable features, such as bumper airbag and hood leading edge airbag. Also, for popup hood and windshield airbag designs, the integration of active sensing signals would greatly enhance their performances (McCarthy and Simmons 2005). 


\section{Countermeasure Evaluations}

\section{Euro NCAP Effects}

The designs of pedestrian-friendly vehicles have been driven by both regulation and consumer test programs. The Euro NCAP pedestrian test, as an example, started in 1997 based on an EEVC test procedure, and has become the benchmark test for evaluating the passive countermeasures for pedestrian protection. In 1997, 30\% of tested vehicles received one star and 70\% received two stars in Euro NCAP pedestrian tests, while in 2007 the distribution changed to $13 \%$ one star, $65 \%$ two stars, and $19 \%$ three stars. Furthermore, since the point score system was introduced in 2009, significant point increases were found (Strandroth et al. 2011). These clear trends show that countermeasures targeting pedestrian protection can be effective. However, correlation between the Euro NCAP ratings and real-world pedestrian injury outcomes has not been established until very recently.

Based on recent Swedish pedestrian injury data and Euro NCAP test results, Strandroth et al. (2011) found that the risk of serious consequences for two-star vehicles was 17 to $38 \%$ lower than for one-star vehicles. Using the GIDAS database and Euro NCAP test results, Liers (2009) estimated that more than $20 \%$ of lower-extremity injuries and $10 \%$ of head injuries could be avoided if all vehicles could achieve 24 points. A later study by the same group (Liers 2011) confirmed the previous finding, but pointed out that the real-world benefit may vary considerably within a given Euro NCAP point level. Furthermore, even with the full points in Euro NCAP pedestrian tests, the serious pedestrian injury risk is still beyond an acceptable extent, indicating a necessity of combining active safety and passive safety designs for pedestrian protection.

\section{Benefit Estimation for Passive and Active Countermeasures}

Current pedestrian test programs, such as the Euro NCAP test program, can provide relatively easy and direct evaluations of passive pedestrian-injury countermeasures without an active sensing system. However, to evaluate the effectiveness of the pop-up hood, windshield airbag, and BASs in reducing pedestrian injuries, whole-body dummy/cadaver tests, computational simulations, or real-world pedestrian crash data are necessary. Even though such data are not extensively available, 
numerous studies have been conducted to estimate the benefit of various passive and active countermeasures for pedestrian protection, given certain assumptions.

By using the GIDAS database, assuming 100\% accuracy of an active sensing system, and a speed reduction of less than $20 \mathrm{~km} / \mathrm{h}$, Rosen et al. (2010) estimated that the effectiveness of an autonomous braking system is about $40 \%$ for reducing pedestrian fatal injuries, and $27 \%$ for serious injuries. Barrios et al. (2009) conducted reconstructions on 140 pedestrian crashes and found that BASs can reduce the impact speed about 5 to 7 $\mathrm{km} / \mathrm{h}$, while autonomous braking with a vision sensor can reduce the impact speed by more than half for most crashes. Whole-body dummy tests and computational humanmodel simulations have also been used to evaluate the effectiveness of an autonomous braking system with a stereo camera (Matsui et al. 2011). The reported impact-velocity reduction ranged from 10 to $15 \mathrm{~km} / \mathrm{h}$, and human-model simulation results showed that this speed reduction is enough to significantly reduce pedestrian head and chest injuries.

To estimate the benefits of active, passive, and integrated active and passive safety systems in reducing pedestrian upper body injuries, Fredriksson et al. (2011) performed simulations with a whole-body pedestrian dummy model and various vehicle front-end geometries. The results indicate that an autonomous braking system could reduce the chest force by $20 \%$ and HIC by $82 \%$, while the pop-up hood and windshield airbag together could reduce the cumulative strain damage measure (CSDM), a brain injury predictor, by $20 \%$, and HIC by $58 \%$. If the active and passive system were combined, they achieved an average reduction of CSDM by $56 \%$ and HIC by $85 \%$. A similar study was conducted by the same group based on GIDAS pedestrian injury data (Fredriksson and Rosen 2012). Effectiveness values of 34\%, 44\%, and 64\% were estimated for passive, active, and integrated countermeasures for reducing pedestrian head injuries, respectively. Both of the above studies demonstrated the benefits of integrating the active and passive safety systems. However, these findings were limited to passenger cars and mini-vans only; pedestrian-to-SUV crashes were not included.

\section{Factors Affecting the Benefits from Countermeasures for Pedestrian Protection}

Even though previous studies have demonstrated clear benefits of design features specific for pedestrian protection, when implementing these features into real vehicles, 
various design features may interact with each other, leading to complex effects. Moreover, vehicles vary significantly in front-end geometry, especially across different vehicle types (passenger cars versus SUVs) and pedestrians vary significantly in stature, weight, age, and other physical conditions. These factors not only substantially influence the benefits of countermeasures for a specific vehicle, but their distributions will also affect the overall benefit estimation for the pedestrian population.

\section{Interactions among Pedestrian Safety Designs}

Active and passive pedestrian-safety designs may interact with each other if implemented together, causing major effects on pedestrian protection. For example, an active precrash brake system will likely result in a pitch angle and a reduced speed of the vehicle right before the impact. Consequently, the pedestrian impact locations, especially secondary head-impact locations, may change for pedestrian-to-vehicle crashes. Interestingly, the pitch angle would move the impact location more rearward, while the decreased impact speed would cause the impact location to move forward with respect to the vehicle front-end structures. This impact-location change will possibly move a head impact in or out of an area protected by the passive safety designs, resulting in significantly different benefit estimations (Fredriksson and Rosen 2012). In spite of such interactive effects between active and passive safety designs, the effectiveness of active safety systems generally requires an adequate passive safety system, and consequently an integrated passive and active system is recommended (Hamacher et al. 2011).

Passive safety designs alone also interact with each other. A lower bumper or a bumper stiffener can effectively reduce the lateral bending of the knee, thus reducing knee injuries, but such designs would potentially increase the head-impact speed (Fowler and Haris 1982), which demands better pedestrian head protection. Computational simulations conducted by Hamacher et al. (2011) showed that a windshield airbag is able to improve adult pedestrian safety significantly, but a pop-up hood can have a negative effect if no windshield airbag is available.

The interacting effects caused by different pedestrian safety designs indicate the necessity of evaluating these designs as a whole rather than as separated systems. Fullscale dummy tests and computational simulations should be able to serve as the tools for 
the integrated system evaluation in addition to the currently available pedestrian test procedures.

\section{Vehicle Types}

The effect of vehicle type on pedestrian injuries has been discussed in the previous sections. LTVs cause significantly higher fatality and injury risks for pedestrians than cars. However, this effect has not been addressed properly by the current pedestrian impact tests, leading to very limited pedestrian safety designs for LTVs. Presumably, safety designs for cars, such as pop-up hood and windshield airbags, should work for LTVs as well. This is true for reducing the overall stiffness of vehicle front-end structures using component tests, but the higher center of gravity of a typical LTV may result in different pedestrian trajectories than those with a car, which can significantly change the benefits of pedestrian safety designs. For example, pedestrian injury data have shown that pedestrians tend to be thrown forward or knocked down by LTVs, whereas they tend to be carried by cars. Therefore, LTVs' hood, hood leading edge, and the ground cause the majority of pedestrian injuries rather than the windshield, which causes the majority of pedestrian injuries for cars. As a result, pop-up hood and hood leading-edge designs will be more important than the windshield airbag for LTVs, which is opposite to the situation for cars. At the same time, because the ground accounts for more injuries for LTVs than for cars, and passive safety designs do not affect the ground impact, active safety designs would be more effective for LTVs than for cars. Furthermore, LTVs cause significantly higher risks of torso injuries than cars, and current pedestrian tests and passive safety designs do not target pedestrian torso protection. Consequently, active safety designs will play a major role in reducing torso injuries in pedestrian-to-LTV crashes. Future pedestrian tests considering torso protection for LTVs would likely motivate more passive safety designs for pedestrian torso protection.

\section{Pedestrian Age}

As discussed in the previous sections, age is one of the most important factors affecting pedestrian injury risks, and consequently pedestrian safety designs will benefit people of different ages very differently. Computer simulations have shown that adults benefit greatly from pop-up hoods and windshield airbags, while children, because of 
their smaller stature, benefit more from active safety designs (Hamacher et al. 2011). Older pedestrians tend to sustain more lower-extremity and chest injuries than younger adults mainly due to the differences in bone strength. As a result, older pedestrian protection needs more stringent requirements for lower-extremity tests and consideration of chest impact tests. These requirements will likely lead to softer, smoother, and more energy-absorbing bumper and hood leading-edge designs, or even bumper/hood leading edge airbag designs. On the other hand, the benefits from a pop-up hood and windshield airbag will be limited for older pedestrians.

\section{Future Trends}

In spite of the complexity involved in developing active safety systems and the limited use of such systems currently, active safety designs may eventually provide the ultimate solution for pedestrian protection by preventing all pedestrian crashes. Passive safety designs, on the other hand, are generally more specific for different body regions, but more reliable and cost-effective. The effectiveness of current active safety systems generally requires an adequate passive safety system, and the interactive effects between these two systems suggest that an integrated system would be necessary to take advantage of both systems.

The increased proportion of SUVs in the vehicle fleet and the increased proportion of elderly in the population would likely demand more safety features specifically designed for SUVs and older populations. In addition to protecting the head and lower-extremities, these design features need to protection the pedestrian torso, which is one of the major concerns in older-pedestrian-to-SUV crashes. Efforts toward developing/modifying pedestrian test procedures specific for SUVs and quantifying the injury-tolerance decrease of older population are also necessary. 


\section{Conclusions}

With the aim of providing insights into vehicle design and new vehicle-safety devices for improving pedestrian safety, a literature review was conducted on pedestrian injuries, pedestrian impact testing, safety features designed for reducing these injuries, and benefit estimations for passive and active pedestrian-safety design features.

\section{Pedestrian Injury Distribution, Causations, and Risk Factors}

Heads and lower extremities are the most commonly injured body regions in vehicle-to-pedestrian crashes, but chest injuries are the second most common injuries after head injuries to cause fatalities. The bumper is the leading cause of pedestrian lower-extremity injuries, while the hood and windshield are the most common cause of pedestrian head injuries. Pedestrian injuries are strongly related to impact speed, pedestrian age, and vehicle type. The increased proportion of older pedestrians and SUVs will likely result in more future pedestrian injuries, especially those involving the torso (chest, abdomen, and spine).

\section{Pedestrian Safety Designs and Benefit Estimation}

Adding energy absorbing materials to the vehicle front-end structures is the most cost-effective way to improve pedestrian safety. However, such improvements often conflict with other design considerations, such as styling, aerodynamics, and other safety standards for low-speed crash tests and rollovers. Deployable passive safety designs, such as pop-up hoods and windshield airbags, have demonstrated considerable benefits in reducing pedestrian head injuries. Active safety designs, such as BASs and autonomous braking systems, although not yet widely implemented, show great potentials for reducing pedestrian injuries. Integrated passive and active systems are recommended for a further enhancement of pedestrian protection.

The benefits from different pedestrian safety designs vary with different types of vehicles and pedestrians with different statures and ages. Consequently it is important to consider implementing specific safety designs to a specific vehicle for maximizing the effectiveness, and population-age profile may also play an important role in selecting pedestrian safety features. 


\section{References}

AAAM (2008). "The Abbreviated Injury Scale (AIS) 2005 - Update 2008." Association for Advancement of Automotive Medicine.

Anderson, R. W., A. J. McLean, et al. (1997). "Vehicle travel speeds and the incidence of fatal pedestrian crashes." Accid Anal Prev 29(5): 667-74.

Ashton, S. J. and Mackay, G. M. (1979). Some characteristics of the population who suffer trauma as pedestrians when hit by cars and some resulting implications. IRCOBI Conference. Gothenburg, Sweden.

Barrios, J. M., Aparicio, A., et al. (2009). Evaluation Of The Effectiveness Of Pedestrian Protection Systems Through In-Depth Accident Investigation, Reconstruction And Simulation. The 21st International Technical Conference on the Enhanced Safety of Vehicles Conference. Stuttgart, Germany.

Belingardi, G., Scattina, A., et al. (2009). Development Of An Hybrid Hood To Improve Pedestrian Safety In Case Of Vehicle Impact. The 21st International Technical Conference on the Enhanced Safety of Vehicles Conference. Stuttgart, Germany.

Carter, E. L., Neal-Sturgess, C. E., et al. (2008). "APROSYS in-depth database of serious pedestrian and cyclist impacts with vehicles." International Journal of Crashworthiness 13(6): 629-642.

Commission, E. (2003). "Directive 2003/102/EC of the European Parliament and of the Council of 17 November 2003 relating to the protection of pedestrians and other vulnerable road users before and in the event of a collision with a motor vehicle and amending Council Directive 70/156/EEC." Official Journal of the European Union, L321: 15-25.

Crandall, J., Bhalla, K. S., et al. (2002). "Designing road vehicles for pedestrian protection." BMJ 324: 1145-8.

Cuerden, R., Richards, D., et al. (2007). Pedestrians And Their Survivability At Different Impact Speeds. The 20th International Technical Conference on the Enhanced Safety of Vehicles Conference. Lyon, France. 
Davis, G. A. (2001). "Relating severity of pedestrian injury to impact speed in vehiclepedestrian crashes - Simple threshold model." 2001 TRB Distinguished Lecture, Pt 1 - Bicycle and Pedestrian Research, Pt 2(1773): 108-113.

Demetriades, D., Murray, J., et al. (2004). "Pedestrians injured by automobiles: relationship of age to injury type and severity." J Am Coll Surg 199(3): 382-7.

Desapriya, E. and Pike, I. (2005). "Sports utility vehicles and older pedestrians: achieving compatibility in motor vehicle crashes." BMJ 331(7522): 966-7.

Desapriya, E., Subzwari, S., et al. (2010). "Do Light Truck Vehicles (LTV) Impose Greater Risk of Pedestrian Injury Than Passenger Cars? A Meta-analysis and Systematic Review." Traffic Injury Prevention 11(1): 48-56.

Eby, D. W. and Molnar, L. J. (2012). Has the Time Come for An Older Driver Vehicle? (Report No. UMTRI-2012-5). University of Michigan Transportation Research Institute. Ann Arbor, MI.

Euro NCAP (2012a). European New Car Assessment Programme - Assessment Protocol - Pedestrian Protection. www.euroncap.com.

Euro NCAP (2012b). European New Car Assessment Programme - Pedestrian Testing Protocol. www.euroncap.com.

Evrard, B. (2011). Innovative Bonnet Active Actuator (B2A) For Pedestrian Protection. The 21st International Technical Conference on the Enhanced Safety of Vehicles Conference. Stuttgart, Germany.

Fildes, B., Gabler, H. C., et al. (2004). Pdestrian Impact Priorities Using Real-World Crash Data and HARM. IRCOBI Conference. Graz, Austria.

Fisher, A. J. and Hall, R. R. (1972). "The influence of car frontal design on pedestrian accident trauma." Accident Analysis and Prevention 4(1): 47-58.

Fowler, J. and Haris, J. (1982). Practical Vehicle Design for Pedestrian Protection. Ninth International Conference on Experimental Safety Vehicles. Kyoto, Japan. 
Fredriksson, R. and Rosen, E. (2012). "Integrated pedestrian countermeasures - Potential of head injury reduction combining passive and active countermeasures." Safety Science 50(3): 400-407.

Fredriksson, R., Rosen, E., et al. (2010). "Priorities of pedestrian protection--a real-life study of severe injuries and car sources." Accid Anal Prev 42(6): 1672-81.

Fredriksson, R., Shin, J., et al. (2011). "Potential of Pedestrian Protection Systems-A Parameter Study Using Finite Element Models of Pedestrian Dummy and Generic Passenger Vehicles." Traffic Injury Prevention 12(4): 398-411.

Fredriksson, R., Håland, Y., et al. (2001). "Evaluation Of A New Pedestrian Head Injury Protection System With A Sensor In The Bumper And Lifting Of The Bonnet's Rear Part." SAE2001-06-0089. Society of Automotive Engineers. Warrendale, PA.

Hamacher, M., Eckstein, L., et al. (2011). Assessment Of Active And Passive Technical Measures For Pedestrian Protection At The Vehicle Front. The 22nd International Technical Conference on the Enhanced Safety of Vehicles. Washington, D.C.

Hannawald, L. and Kauer, F. (2004). Equal Effectiveness Study on Pedestrian Protection. Technische Universität Dresden. Dresden, Germany.

Hardy, B. J., Lawrence, G. J. L., et al. (2006). A Study on the Feasibility of Measures Relating to the Protection of Pedestrians and Other Vulnerable Road Users. Project Report UPR/VE/045/. TRL Limited, Crowthorne, U.K.

Helmer, T., Ebner, A., et al. (2010). "Injury risk to specific body regions of pedestrians in frontal vehicle crashes modeled by empirical, in-depth accident data." Stapp Car Crash J 54: 93-117.

Henary, B. Y., Ivarsson, J., et al. (2006). "The influence of age on the morbidity and mortality of pedestrian victims." Traffic Inj Prev 7(2): 182-90.

Hu, J., Chou, C. C., et al. (2007). "A weighted logistic regression analysis for predicting the odds of head/face and neck injuries during rollover crashes." Annu Proc Assoc Adv Automot Med 51: 363-79. 
Huang, S. and Yang, J. (2010). "Optimization of a reversible hood for protecting a pedestrian's head during car collisions." Accid Anal Prev 42(4): 1136-43.

Inomata, Y., Iwai, N., et al. (2009). Development Of The Pop-Up Engine Hood For Pedestrian Head Protection. The 21st International Technical Conference on the Enhanced Safety of Vehicles Conference. Stuttgart, Germany.

Ivarsson, B. J., Henary, B., et al. (2005). "Significance of adult pedestrian torso injury." Annu Proc Assoc Adv Automot Med 49: 263-77.

Kajzer, J., Matsui, Y., et al. (1999). "Shearing and bending effects at the knee joint at low-speed lateral loading." SAE1999-01-0712. Society of Automotive Engineers. Warrendale, PA.

Kajzer, J., Schroeder, G., et al. (1997). Shearing and bending effects at the knee joint at high-speed lateral loading. 41st Stapp Car Crash Conference. Lake Buena Vista, FL.

Kalliske, I. and Friesen, F. (2001). Improvements to Pedestrian Protection as Exemplified on a Standard-Sized Car. The 17th International Technical Conference on the Enhanced Safety of Vehicles. Amsterdam, The Netherlands.

Kent, R., Henary, B., et al. (2005). "On the fatal crash experience of older drivers." Annu Proc Assoc Adv Automot Med 49: 371-91.

Kent, R., Lee, S. H., et al. (2005). "Structural and material changes in the aging thorax and their role in crash protection for older occupants." Stapp Car Crash J 49: 23149.

Kent, R., Trowbridge, M., et al. (2009). "How many people are injured and killed as a result of aging? Frailty, fragility, and the elderly risk-exposure tradeoff assessed via a risk saturation model." Ann Adv Automot Med 53: 41-50.

Kerkeling, C., Schäfer, J., et al. (2005). Structural Hood And Hinge Concepts For Pedestrian Protection. The 19th International Technical Conference on the Enhanced Safety of Vehicles. Washington, D.C. 
Kim, J. K., Ulfarsson, G. F., et al. (2008). "Age and pedestrian injury severity in motorvehicle crashes: a heteroskedastic logit analysis." Accid Anal Prev 40(5): 1695702.

Klinich, K. D. and Schneider, L. W. (2003). Biomechanics of Pedestrian Injuries Related to Lower Extremity Injury Assessment Tools: A Review of the Literature and Analysis of Pedestrian Crash Database. (Report No. UMTRI-2003-25). University of Michigan Transportation Research Institute. Ann Arbor, MI.

Kong, C. and Yang, J. (2010). "Logistic regression analysis of pedestrian casualty risk in passenger vehicle collisions in China." Accid Anal Prev 42(4): 987-93.

Kuehn, M., Froeming, R., et al. (2005). Assessment of Vehicle Related Pedestrian Safety. The 19th International Technical Conference on the Enhanced Safety of Vehicles. Washington, D.C.

Laituri, T. R., Prasad, P., et al. (2005). Derivation and Evaluation of a Provisional, AgeDependent, AIS3+ Thoracic Risk Curve for Belted Adults in Frontal Impacts. SAE2005-01-0297. Society of Automotive Engineers. Warrendale, PA.

Lee, C. and Abdel-Aty, M. (2005). "Comprehensive analysis of vehicle-pedestrian crashes at intersections in Florida." Accid Anal Prev 37(4): 775-86.

Lee, K. B., Jung, H. J., et al. (2007). The Study On Developing Active Hood Lift System For Decreasing Pedestrian Head Injury. The 20th International Technical Conference on the Enhanced Safety of Vehicles Conference. Lyon, France.

Lefler, D. E. and Gabler, H. C. (2004). "The fatality and injury risk of light truck impacts with pedestrians in the United States." Accid Anal Prev 36(2): 295-304.

Li, G., Braver, E. R., et al. (2003). "Fragility versus excessive crash involvement as determinants of high death rates per vehicle-mile of travel among older drivers." Accid Anal Prev 35(2): 227-35.

Liers, H. (2009). Benefit Estimation of the Euro NCAP Pedestrian Rating Concerning Real World Pedestrian Safety. The 21st International Technical Conference on the Enhanced Safety of Vehicles Conference. Stuttgart, Germany. 
Liers, H. (2011). Benefit Estimation of secondary Safety Measures in Real-World Pedestrian Accidents. The 22nd International Technical Conference on the Enhanced Safety of Vehicles Conference. Washington, D.C.

Liu, Q., Xia, Y., et al. (2009). Design Analysis Of A Sandwich Hood Structure For Pedestrian Protection. The 21st International Technical Conference on the Enhanced Safety of Vehicles Conference. Stuttgart, Germany.

Longhitano, D., Henary, B., et al. (2005). "Influence of Vehicle Body Type on Pedestrian Injury Distribution." SAE2005-01-1876. Society of Automotive Engineers. Warrendale, PA.

Martin, J. L., Lardy, A., et al. (2011). "Pedestrian injury patterns according to car and casualty characteristics in france." Ann Adv Automot Med 55: 137-46.

Matsui, Y. (2005). "Effects of vehicle bumper height and impact velocity on type of lower extremity injury in vehicle-pedestrian accidents." Accid Anal Prev 37(4): $633-40$.

Matsui, Y., Han, Y., et al. (2011). "Performance of Collision Damage Mitigation Braking Systems and Their Effects on Human Injury in the Event of Car-to-Pedestrian Accidents." Stapp Car Crash J 55: 461-478.

Matsui, Y., Hitosugi, Y., et al. (2011). "Severity of vehicle bumper location in vehicle-topedestrian impact accidents." Forensic Science International 212(1-3): 205-209.

McCarthy, M. and Simmons, I. (2005). Active Pedestrian Protection. The 19th International Technical Conference on the Enhanced Safety of Vehicles. Washington, D.C.

Mizuno, Y. (2005). Summary Of IHRA Pedestrian Safety WG Activities (2005) Proposed Test Methods To Evaluate Pedestrian Protection Afforded By Passenger Cars. The 19th International Technical Conference on the Enhanced Safety of Vehicles. Washington, D.C.

Morris, A., Welsh, R., et al. (2002). "An overview of requirements for the crash protection of older drivers." Annu Proc Assoc Adv Automot Med 46: 141-56. 
Naci, H., Chisholm, D., et al. (2009). "Distribution of road traffic deaths by road user group: a global comparison." Inj Prev 15(1): 55-9.

Neal-Sturgess, C., Carter, E., et al. (2007). APROSYS European In-Depth Pedestrian Database. The 20th International Technical Conference on the Enhanced Safety of Vehicles Conference. Lyon, France.

Nishimoto, T. (2003). Introduction of the regulation of pedestrian head protection in Japan. . 18th International Technical Conference on the Enhanced Safety of Vehicles. Nagoya, Japan.

Oh, C., Kang, Y. S., et al. (2008). "Assessing the safety benefits of an advanced vehicular technology for protecting pedestrians." Accid Anal Prev 40(3): 935-42.

Oh, C., Kang, Y. S., et al. (2008). "Development of probabilistic pedestrian fatality model for characterizing pedestrian-vehicle collisions." International Journal of Automotive Technology 9(2): 191-196.

Otte, D. and Haasper, C. (2005). Technical Parameters and Mechanisms for The Injury Risk of The Knee Joint of Vulnerable Road Users Impacted by Cars in Road Traffic Accidents. IRCOBI Conference. Prague, Czech Republic.

Otte, D., Jansch, M., et al. (2012). "Injury protection and accident causation parameters for vulnerable road users based on German In-Depth Accident Study GIDAS." Accident Analysis and Prevention 44(1): 149-153.

Pasanen, E. and Salmivaara, H. (1993). "Driving speeds and pedestrian safety in the city of Helsinki." Traffic Eng. Control 34: 308-310.

Paulozzi, L. J. (2005). "United States pedestrian fatality rates by vehicle type." Inj Prev 11(4): 232-6.

Perron, T., Kassaagi, M., et al. (2001). Active safety experiments with common drivers for the specification of active safety systems. The 17th International Technical Conference on the Enhanced Safety of Vehicles. Amsterdam, the Netherlands. 
Pinecki, C., Fontaine, L., et al. (2011). Pedestrian Protection - Physical And Numerical Analysis Of The Protection Offered By The Windscreen. The 22nd International Technical Conference on the Enhanced Safety of Vehicles. Washington, D.C.

Pipkorn, B., Fredriksson, R., et al. (2007). Bumper Bag for SUV to Passenger Vehicle Compatibility and Pedestrian Protection. The 20th International Technical Conference on the Enhanced Safety of Vehicles Conference. Lyon, France.

Richards, D., Cookson, R., et al. (2009). The Causes Of Pedestrians' Head Injuries Following Collisions With Cars Registered In 2000 Or Later. The 21st International Technical Conference on the Enhanced Safety of Vehicles Conference. Stuttgart, Germany.

Rosen, E., Kallhammer, J. E., et al. (2010). "Pedestrian injury mitigation by autonomous braking." Accident Analysis and Prevention 42(6): 1949-1957.

Rosen, E. and Sander, U. (2009). "Pedestrian fatality risk as a function of car impact speed." Accid Anal Prev 41(3): 536-42.

Rosen, E., Stigson, H., et al. (2011). "Literature review of pedestrian fatality risk as a function of car impact speed." Accid Anal Prev 43(1): 25-33.

Roudsari, B. S., Mock, C. N., et al. (2005). "An evaluation of the association between vehicle type and the source and severity of pedestrian injuries." Traffic Inj Prev 6(2): $185-92$.

Roudsari, B. S., Mock, C. N., et al. (2004). "Pedestrian crashes: higher injury severity and mortality rate for light truck vehicles compared with passenger vehicles." Inj Prev 10(3): 154-8.

Schuster, P. J. (2006). "Current Trends in Bumper Design for Pedestrian Impact." SAE2006-01-0464. Society of Automotive Engineers. Warrendale, PA.

Schuster, P. J. and Staines, B. (1998). Determination of Bumper Styling and Engineering Parameters to Reduce Pedestrian Leg Injuries. SAE980361. Society of Automotive Engineers. Warrendale, PA. 
Shin, M. K., Park, K. T., et al. (2008). "Design of the active hood lift system using orthogonal arrays." Proceedings of the Institution of Mechanical Engineers Part D-Journal of Automobile Engineering 222(D5): 705-717.

Simms, C. and O'Neill, D. (2005). "Sports utility vehicles and older pedestrians." BMJ 331(7520): 787-8.

Simms, C. K. and Wood, D. P. (2006). "Pedestrian risk from cars and sport utility vehicles - a comparative analytical study." Proceedings of the Institution of Mechanical Engineers Part D-Journal of Automobile Engineering 220(D8): 10851100.

Snedeker, J. G., Muser, M. H., et al. (2003). "Assessment of pelvis and upper leg injury risk in car-pedestrian collisions: comparison of accident statistics, impactor tests and a human body finite element model." Stapp Car Crash J 47: 437-57.

Snedeker, J. G., Walz, F. H., et al. (2005). Assessing Femur and Pelvis Injury Risk in Car-Pedestrian Collisions: Comparison of Full Body PMTO Impact and a Human Body Finite Element Model. The 19th International Technical Conference on the Enhanced Safety of Vehicles. Washington, D.C.

Strandroth, J., Rizzi, M., et al. (2011). "The Correlation Between Pedestrian Injury Severity in Real-Life Crashes and Euro NCAP Pedestrian Test Results." Traffic Injury Prevention 12(6): 604-613.

Takahashi, Y., Kikuchi, Y., et al. (2000). "Development and validation of the finite element model for the human lower limb of pedestrians." Stapp Car Crash J 44: $335-55$.

Watts, J. S. (2005). "Sports utility vehicles and older pedestrians: not all SUVs are the same." BMJ 331(7522): 967.

WHO (2009). "Global Status Report on Road Safety." World Health Organization. Geneva, Switzerland.

Xu, J., Li, Y. B., et al. (2011). "Automotive windshield - pedestrian head impact: Energy absorption capability of interlayer material." International Journal of Automotive Technology 12(5): 687-695. 
Yaksich, S. J. (1964). "Pedestrians with Milage: A Study of Elderly Pedestrian Accidents in St. Petersburg, Florida." American Automobile Association. Washington, D.C.

Yang, J. (2005). "Review of injury biomechanics in car-pedestrian collisions." Int. J. Vehicle Safety 1(1/2/3): 100-117.

Zegeer, C. V. and Bushell, M. (2012). "Pedestrian crash trends and potential countermeasures from around the world." Accid Anal Prev 44(1): 3-11.

Zhang, G., Cao, L., et al. (2008). "A field data analysis of risk factors affecting the injury risks in vehicle-to-pedestrian crashes." Ann Adv Automot Med 52: 199-214.

Zhao, H., Yin, Z. Y., et al. (2010). "Investigation of 184 passenger car-pedestrian accidents." International Journal of Crashworthiness 15(3): 313-320.

Zhou, Q., Rouhana, S. W., et al. (1996). Age Effects on Thoracic Injury Tolerance. The 40th STAPP Car Crash Conference. Minneapolis, MN. 